Research Article

\title{
The Investigation of a Wealth Distribution Model on Isolated Discrete Time Domains
}

\author{
Chunhua Hu $(\mathbb{D}$, Wenyi Huang $(\mathbb{D}$, and Tianhao Xie \\ School of Economic Mathematics, Southwestern University of Finance and Economics, Chengdu 611130, China \\ Correspondence should be addressed to Chunhua Hu; chunhuahu@smail.swufe.edu.cn
}

Received 11 September 2019; Revised 13 December 2019; Accepted 9 January 2020; Published 11 February 2020

Academic Editor: Sajad Azizi

Copyright (C) 2020 Chunhua Hu et al. This is an open access article distributed under the Creative Commons Attribution License, which permits unrestricted use, distribution, and reproduction in any medium, provided the original work is properly cited.

\begin{abstract}
A wealth distribution model on isolated discrete time domains, which allows the wealth to exchange at irregular time intervals, is used to describe the effect of agent's trading behavior on wealth distribution. We assume that the agents have different degrees of risk aversion. The hyperbolic absolute risk aversion (HARA) utility function is employed to describe the degrees of risk aversion of agents, including decreasing relative risk aversion (DRRA), increasing relative risk aversion (IRRA), and constant relative risk aversion (CRRA). The effect of agent's expectation on wealth distribution is taken into account in our wealth distribution model, in which the agents are allowed to adopt certain trading strategies to maximize their utility and improve their wealth status. The Euler equation and transversality condition for the model on isolated discrete time domains are given to prove the existence of the optimal solution of the model. The optimal solution of the wealth distribution model is obtained by using the method of solving the rational expectation model on isolated discrete time domains. A numerical example is given to highlight the advantages of the wealth distribution model.
\end{abstract}

\section{Introduction}

In recent years, unequal distribution of wealth has caused a series of negative phenomena in the society. Empirical and theoretical research on wealth distribution has become an active branch of modern economics. The problem of wealth distribution is closely related to the optimal conditions of economic growth. Studying the distribution of wealth is important for analyzing the wealth inequality and formulating tax and redistributive policies.

The dynamic distribution of wealth is a complex phenomenon, which is determined by many factors, including tax, welfare, innovation, education, consumption, and agents' preferences. Guala [1] studies the redistribution of wealth among agents in the presence of taxation in the trading process. Pareschi and Toscani [2] consider the influence of agent's knowledge level on wealth distribution and analyze the formation process of knowledge. Toscani et al. [3] discuss the influence of agents' preferences on wealth distribution and get Pareto curve of wealth distribution. Brugna and Toscani [4] generalize the works of Toscani et al.
[3] and further investigate the changes in wealth distribution of two types of agents with different trading strategies. A. S. Chakrabarti and B. K. Chakrabarti [5] propose a model based on consumers' optimization which can give rise to those particular forms of asset exchange equations and discuss a problem of income distribution. In [6], the authors further investigate income and wealth inequality in the context of a generalized kinetic exchange model and conclude that the economy indeed shows inequality reversal under some certain conditions. Chakrabarti [7] discusses the laws of wealth distribution, firm size distribution, and the city size distribution in a common framework and shows that the equilibrium configurations of some general economic mechanisms are consistent with a power law in general and Zipf s law in particular, in size distribution. A survey of wealth distribution is referred to [8].

In fact, wealth distribution is not only related to the knowledge level and preference of agents but also to the transaction time. Therefore, it is necessary to divide the agent's transaction into several periods and set up a discrete wealth distribution model. Chatterjee et al. [9] assume that 
the agent's saving tendency satisfies a certain distribution and give a discrete wealth distribution model. Benhabib et al. [10] investigate the wealth distribution in Bewley economies with capital income risk and solve an infinite horizon consumption-saving problem with incomplete markets. In [10], the equilibrium distribution for consumption, savings, and wealth is obtained under the assumption that agents face saving restrictions. Mariacristina and Giulio [11] study the influence of agent's savings on wealth distribution and find out the factors causing wealth inequality. Other discrete models on wealth distribution can be found in [12-16].

In actual transactions, agents usually anticipate the price of assets or commodities in the market based on past trading information. This anticipated behavior affects the distribution of wealth among agents. Acedńnski [17] discusses the influence of agent's expectation on wealth distribution. The results show that the effect of agent's expectation on wealth distribution is obvious. In [17], a standard constant relative risk aversion (CRRA) utility function is used to describe the behaviors of agents, and a discrete wealth distribution model affected by agent's expectation is given. In $[10,17]$, although the agent's transaction is divided into several periods, the time interval of the transaction is uniform, without considering the wealth distribution when the transaction interval is uneven.

Generally speaking, trading is not a uniformly discrete nor continuous behavior. Consider the stock market as an example. In a stock market, an agent buys shares of a particular company in varying time intervals. Suppose that on the first week of the year, the agent buys stocks every day, and in the next several weeks, the company generates a good profit, which makes the agent buy stocks several times a week. Unfortunately, due to the company's poor performance and lower earnings for the rest of the year, the agent buys only one stock on the last week of the year. Since there is no rule to restrict the agent's trading behavior, we do not know whether the time interval of trading is uniform. As a result, the agent's trading behavior needs to be described in isolated discrete time domains where events may occur at unevenly spaced time points. Atici et al. [18] present a dynamic optimal problem from economics and construct a time scale model. Atici et al. [19] investigate a perfectforesight utility maximisation problem by using a method for solving the dynamic optimisation problem in which the real-valued function and constraints depend on different times. Atici and Turhan [20] discuss the deterministic dynamic sequence problem on isolated discrete time domains. Atici et al. [21] introduce a nonlinear stochastic dynamic problem on isolated discrete time domains and give the Euler equation and transversality condition for the problem. The work in [21] provides a method for solving the nonlinear growth model on isolated discrete time domains. Other studies about the isolated discrete models can be found in [22-25].

In $[10,17,21]$, a CRRA utility function is utilized to describe the agent's behavior. In actual transactions, the agent's aversion for risk is complex. To better study the distribution of wealth among agents, we employ the hyperbolic absolute risk aversion (HARA) utility function to characterize the degrees of risk aversion of agents. The HARA utility function includes several agents' aversion for risk, such as decreasing relative risk aversion (DRRA), increasing relative risk aversion (IRRA), and constant relative risk aversion (CRRA). In recent years, the HARA utility function has been applied in various economic models. Achury et al. [26] study a two-asset portfolio choice model with the HARA utility function which includes the subsistence consumption parameter. Levaggi and Menoncin [27] consider optimal dynamic tax evasion with the HARA utility function and a penalty function. Menoncin and Nembrini [28] find a closed form solution for the stochastic continuous time growth model with the HARA utility function.

Some researchers show that the problem of wealth distribution is not only related to the optimal conditions of economic growth but also closely related to the wealth inequality and the formulation of tax policies. To reduce the inequality of wealth distribution, it is very important to analyze the factors that affect wealth distribution, such as the preferences of agents, trading strategies, and trading time. Motivated by the works in $[10,17,21,28]$, we assume that agents estimate the next saving rate and profit of assets based on past trading information. We allow agents to adopt certain trading strategies to maximize their utility, such as using part of the assets for trading and the rest for savings. Under these assumptions, we investigate the influence of agent's trading behavior and average consumption level on the wealth distribution.

The objective of this work is to employ the HARA utility function to describe the agents' behavior and discuss the influence of average consumption level and trading time of agents on wealth distribution in the multiagent market. Compared with the works in $[17,21]$, we utilize the HARA utility function to characterize the agent's behavior and divide the agent's transaction into multiple periods. A wealth distribution model on isolated discrete time domains, which allows the wealth to exchange on unevenly spaced time points, is given. HARA utility function and the wealth distribution model are different from those in $[17,21]$, in which the CRRA utility function is used and the time domain of the wealth distribution model is a uniformly discrete set of points which allows the wealth to exchange at uniform time interval. HARA utility function contains several kinds of utility functions, e.g., DRRA utility function, IRRA utility function, and CRRA utility function. The time domain of the wealth distribution model in our work is a collection of points along the real number, which describes the distribution of wealth in nonuniform time interval. Consequently, we extend parts of results in $[17,21]$.

The study is organized as follows. We introduce the calculus and dynamic equation on isolated discrete time domains in Section 2. The Euler equation and transversality condition of a nonlinear stochastic dynamic model with a bivariate utility function are derived in Section 3. The main results about the wealth distribution model are given in Section 4. In Section 5, a numerical example is given to illustrate the advantages of the wealth distribution model on isolated discrete time domains. 


\section{Calculus and Dynamic Equation on Isolated Discrete Time Domains}

In Sections 3 and 4, we will set up the nonlinear stochastic dynamic model and the wealth distribution model on the isolated discrete time domains. The isolated discrete time domains are different from the classic discrete time domain. The classical discrete time domain contains only uniform time points, while the isolated discrete time domains contain not only uniform time points but also nonuniform time points. Therefore, we will introduce some definitions, theorems, properties, and dynamic equations on isolated discrete time domains.

In this section, the basic concepts about delta-derivative, delta-integral, exponential function, and dynamic equation on isolated discrete time domains are introduced.

Let $\mathbb{T}$ be any nonempty closed subset of the real numbers $\mathbb{R}$. The operators $\sigma(t), p(t)$, and $\mu(t)$ are defined by $\sigma(t)=\inf \{s \in \mathbb{T}: s>t\}, \quad p(t)=\sup \{s \in \mathbb{T}: s<t\}, \quad$ and $\mu(t)=\sigma(t)-t$, respectively, where $\sigma\left(t_{i}\right)$ is the forward jump operator, $\mu\left(t_{i}\right)$ is the forward graininess operator, and $p\left(t_{i}\right)$ is the backward jump operator. From the time scale $\mathbb{T}$, the set $\mathbb{T}^{k}$ is obtained. For a point $t \in \mathbb{T}$ if $\sigma(t)>t$, we say that $t$ is right-scattered, and if $p(t)<t$, we say that $t$ is left-scattered. When $\mathbb{T}$ has a left-scattered maximum $m$, we have $\mathbb{T}^{k}=\mathbb{T}-\{m\}$. Otherwise, $\mathbb{T}^{k}=\mathbb{T}$.

It is worth noting that, in the case $\mathbb{T}=\mathbb{R}$, we obtain (see Merrell et al. [29])

$$
\begin{aligned}
& \sigma(t)=p(t)=t \\
& \mu(t) \equiv 0 .
\end{aligned}
$$

When $\mathbb{T}=\mathbb{Z}$, where $\mathbb{Z}$ is the set of positive integers, for any $t \in \mathbb{Z}$, we have

$$
\begin{aligned}
& \sigma(t)=t+1, \\
& p(t)=t-1, \\
& \mu(\mathrm{t}) \equiv 1 .
\end{aligned}
$$

When $P^{\mathbb{N}}=\left\{P^{n} \mid n \in \mathbb{N}\right\}$, where $P>1$ and $\mathbb{N}$ is the natural number, we get

$$
\begin{aligned}
\sigma(t) & =P t, \\
p(t) & =\frac{t}{P}, \\
\mu(t) & =(P-1) t .
\end{aligned}
$$

$P^{\mathbb{N}}=\left\{1, P, P^{2}, \ldots\right\}$ is the called quantum time scale, which is very important in quantum theory (see Merrell et al. [29]).

Let $F: \mathbb{T} \longrightarrow \mathbb{R}$ be a real-valued function on $\mathbb{T}$. The delta-derivative $[21,30]$ of $F$ is denoted by

$$
F^{\Delta}(t)=\frac{F(\sigma(t))-F(t)}{\mu(t)},
$$

where $t \in \mathbb{T}$. The delta-integral $[21,30]$ of $F$ is defined by

$$
\int_{0}^{T} F(\tau) \Delta \tau=\sum_{s \in[0, T) \cap \mathbb{T}} \mu(s) F(s),
$$

and the exponential function $[21,25,30]$ is given as

$$
e_{q}\left(t, t_{0}\right)=\prod_{s \in\left[t_{0}, t\right) \cap \mathbb{T}}(1+\mu(s) q(s)),
$$

where $1+q(t) \mu(t) \neq 0$ for all $t \in \mathbb{T}$.

We mention that when $\mathbb{T}=\mathbb{Z}$, exponential function $e_{q}\left(t, t_{0}\right)$ becomes

$$
e_{q}\left(t, t_{0}\right)=(1+q)^{t-t_{0}}
$$

where $1+q \neq 0$.

When $\mathbb{T}=P^{\mathbb{Z}}$, we get

$$
e_{q}\left(t, t_{0}\right)=\prod_{s \in\left[t_{0}, t\right) \cap \mathbb{T}}[1+(P-1) q s], \quad t>t_{0} .
$$

For exponential function $e_{(q-1) / \mu}\left(t, t_{0}\right)$, we have

$$
e_{(q-1) / \mu}\left(t, t_{0}\right)=\left\{\begin{array}{cc}
\prod_{s \in\left[t_{0}, t\right) \cap \mathbb{T}} q(s), & t \geq t_{0}, \\
\prod_{s \in\left[t_{0}, t\right) \cap \pi} \frac{1}{q(s)}, & t<t_{0} .
\end{array}\right.
$$

Let $0<q<1$ be a constant and $n_{t}$ be a function of $t$, where $n_{t}$ indicates that the number of discrete points on the interval $\left[t_{0}, t\right)$. Exponential function $e_{(q-1) / \mu}\left(t, t_{0}\right)$ is rewritten by

$$
e_{(q-1) / \mu}\left(t, t_{0}\right)=\prod_{s \in\left[t_{0}, t\right] \cap \mathbb{T}} q(s)=q^{n_{t}},
$$

where

$$
n_{t}(t, s):=\int_{s}^{t} \frac{\Delta(\tau)}{\mu(\tau)}
$$

To calculate the wealth distribution model in Section 4, we introduce an algorithm on time scales.

Lemma 1 (see [30]). Let $Q$ be a $n \times n$-matrix. If $\lambda_{1}, \lambda_{2}, \lambda_{3}, \ldots$, $\lambda_{n}$ are the eigenvalues of $Q$, then

$$
Q^{t}=\sum_{i=0}^{n-1} r_{i+1}(t) U_{i}
$$

where $r_{i}(t)(i=1,2, \ldots, n)$ are chosen to satisfy the system

$$
\begin{aligned}
{\left[\begin{array}{c}
r_{1}(t+1) \\
r_{2}(t+1) \\
\vdots \\
r_{n}(t+1)
\end{array}\right] } & =\left[\begin{array}{ccccc}
\lambda_{1} & 0 & 0 & \cdots & 0 \\
1 & \lambda_{2} & 0 & \cdots & 0 \\
0 & 1 & \lambda_{3} & \cdots & 0 \\
\vdots & \cdots & \cdots & \cdots & \vdots \\
0 & \cdots & 0 & 1 & \lambda_{n}
\end{array}\right]\left[\begin{array}{c}
r_{1}(t) \\
r_{2}(t) \\
\vdots \\
r_{n}(t)
\end{array}\right], \\
{\left[\begin{array}{c}
r_{1}(0) \\
r_{2}(0) \\
r_{3}(0) \\
\vdots \\
r_{n}(0)
\end{array}\right] } & =\left[\begin{array}{c}
1 \\
0 \\
0 \\
\vdots \\
0
\end{array}\right],
\end{aligned}
$$


and $U_{i}$ are defined by

$$
\begin{aligned}
& U_{0}=I, \\
& U_{i}=\left(Q-\lambda_{i} I\right) U_{i-1}, \quad(1 \leq i \leq n) .
\end{aligned}
$$

The algorithm in Lemma 1 is called Putzer algorithm. The application of Lemma 1 is displayed in the numerical example.

Definition 1 (see [30]). If $h \in \mathscr{R}$ and $f: \mathbb{T} \longrightarrow \mathbb{R}$ is the rightdense continuous function, then the dynamic equation

$$
y^{\Delta}(t)=h(t) y(t)+f(t)
$$

is called regressive.
Definition 2 (see [30]). Let $h: \mathbb{T} \longrightarrow \mathbb{R}$ be the right-dense continuous function. Then, the circle minus of $h(t)$ for all $t \in \mathbb{T}^{k}$ is denoted by

$$
\Theta h(t):=-\frac{h(t)}{1+\mu(t) h(t)}
$$

where $1+\mu(t) h(t) \neq 0, h \in \mathscr{R}$ is the set of all regressive.

Lemma 2 (see [21]). Let $Y_{t}$ be an $n \times 1$ vector function on isolated discrete time domains and satisfy

$$
Y_{t}=A E_{t}\left[Y_{t}^{\sigma}\right]+F\left(t, Z_{t}\right)
$$

where $A$ is the $n \times n$ nonsingular matrix and $F$ is the $n \times 1$ vector function. Then, the solution of equation (17) is

$$
Y_{t}=e_{(I-A) A^{-1}(1 / \mu)}(t, 0) M(t)-e_{(I-A) A^{-1}(1 / \mu)}(t, 0) \int_{0}^{T} e_{\Theta(I-A) A^{-1}(1 / \mu)}(s, 0) \frac{1}{\mu(s)} F\left(s, Z_{s}\right) \Delta s
$$

where $t \in \mathbb{T}$ and $M(t)$ is a $n \times n$ arbitrary matrix martingale on $\mathbb{T}$ and meets the martingale property $E_{t}\left[M^{\sigma}(t)\right]=M(t)$.

For more information of dynamic equations on isolated discrete time domains, see [30].

\section{Nonlinear Stochastic Dynamic Model with a Bivariate Utility Function}

We assume that the isolated discrete time domains $\mathbb{t}$ satisfy sup $\mathbb{T}=\infty$. Let $\mathbb{T} \cap[0, \infty)=[0, \infty)$. Then, the nonlinear stochastic dynamic model (NSDM) with a bivariate utility function on $\mathbb{T}$ is given by

$$
\begin{aligned}
& \sup _{\left\{c_{i, t}, a_{i, t}\right\}_{t=0}^{\infty}} E_{0} \sum_{t \in[0, \infty) \mathbb{T}} e_{\left(\beta_{i}-1\right) / \mu}(t, 0) U\left(c_{i, t}, \bar{c}_{t}\right) \mu(t) \\
& \text { s.t. }\left\{\begin{array}{l}
c_{i, t}=f\left(a_{i, t}, a_{i, \sigma(t)}, y_{i, \sigma(t)}, r_{i, \sigma(t)}\right), \\
\bar{c}_{t}=f\left(\bar{c}_{t}, \bar{c}_{\sigma(t)}, \bar{y}_{\sigma(t)}, \bar{r}_{\sigma(t)}\right), \\
a_{i, \sigma(0)}, \bar{a}_{\sigma(0)}, c_{i, 0}, \bar{c}_{0} \in X, \quad \text { are given, }
\end{array}\right.
\end{aligned}
$$

where $0<\beta_{i}<1, a_{i, t}(i=1,2, \ldots, n)$, and $\bar{a}_{\sigma(t)}$ are the state variables, $\sigma: \mathbb{T} \longrightarrow \mathbb{T}$ is the forward jump operator, $y_{i, t}(i=1$, $2, \ldots, n), \bar{y}_{\sigma(t)}, r_{i, t}(i=1,2, \ldots, n)$, and $\bar{r}_{\sigma(t)}$ are the random state variables, $E_{0}$ represents the mathematical expectation, $U: \mathbb{R}^{2} \longrightarrow \mathbb{R}^{+}$is a strictly increasing, concave, continuous, and differentiable utility function, $X=\kappa \times \kappa$, where $\kappa=$ $\left\{\left\{x_{t}\right\}_{t=0}^{\infty}: x_{t} \geq 0, t \in[0, \infty)\right\}$, and $f(x, y, z, u): \mathbb{R}^{4} \longrightarrow \mathbb{R}^{+}$is a concave in $(x, y)$, continuous, and differentiable real-valued function with $f_{y} \leq 0$. The discount factor relating to NSDM $(19)$ is $e_{\left(\beta_{i}-1\right) / \mu}(t, 0)$. When $\mathbb{T}=\mathbb{Z}, e_{\left(\beta_{i}-1\right) / \mu}(t, 0)=\beta_{i}^{t}$, where $\beta_{i}^{t}$ is a standard discount factor in the case of uniform time domain.

We mention that the concavity in $(x, y)$ means concavity in two variables $x$ and $y$ jointly, not in each variable separately. The concavity of $f(x, y, z, u)$ is used to find the optimal solution of NSDM (19), namely, function $f(x, y, z, u)$ is concave in $(x, y)$ if and only if for any pair of distinct points $(x, y, z, u)$ and $(\bar{x}, \bar{y}, \bar{z}, \bar{u})$ in the domain, we have

$$
\begin{aligned}
f(x, y, z, u)-f\left(x^{*}, y^{*}, z, u\right) \leq & f_{x}\left(x^{*}, y^{*}, z, u\right)\left(x-x^{*}\right) \\
& +f_{x}\left(x^{*}, y^{*}, z, u\right)\left(y-y^{*}\right),
\end{aligned}
$$

$$
\begin{aligned}
f(\bar{x}, \bar{y}, \bar{z}, \bar{u})-f\left(\overline{x^{*}}, \overline{y^{*}}, \bar{z}, \bar{u}\right) \leq & f_{x}\left(\overline{x^{*}}, \overline{y^{*}}, \bar{z}, \bar{u}\right)\left(\bar{x}-\overline{x^{*}}\right) \\
& +f_{y}\left(\overline{x^{*}}, \overline{y^{*}}, \bar{z}, \bar{u}\right)\left(\bar{y}-\overline{y^{*}}\right) .
\end{aligned}
$$

To find optimal sequence $\left\{c_{i, t}, a_{i, t}\right\}_{t=0}^{\infty}$ to maximize the expected utility in NSDM (19), the Euler equation and transversality condition for NSDM (19) need to be given.

An Euler equation is the first-order condition for describing optimal choice of dynamic stochastic problems. There are several methods for the analysis and definition of Euler equation, namely, calculus of variations, optimal control theory, the Lagrangian method, and dynamic programming $[31,32]$. We assume that NSDM (19) reaches the upper bound at $\left\{c_{i, t}, a_{i, t}\right\}_{t=0}^{\infty}$. The Euler equation for NSDM (19) is defined as

$$
\left\{\begin{array}{l}
\mu_{t} U_{c_{i, t}^{*}}^{\prime} f_{y}\left(a_{i, t}^{*}, a_{i, \sigma(t)}^{*}, y_{i, \sigma(t)}, r_{i, \sigma(t)}\right)+E_{t}\left[\beta_{i} \mu_{\sigma(t)} U_{c_{i, \sigma(t)}^{*}}^{\prime} f_{x}\left(a_{i, \sigma(t)}^{*}, a_{i, \sigma(t+1)}^{*}, y_{i, \sigma(t+1)}, r_{i, \sigma(t+1)}\right]=0,\right. \\
\mu_{t} U_{\bar{c}_{t}^{*}}^{\prime} f_{y}\left(\bar{a}_{t}^{*}, \bar{a}_{\sigma(t)}^{*}, \bar{y}_{\sigma(t)}, \bar{r}_{\sigma(t)}\right)+E_{t}\left[\beta_{i} \mu_{\sigma(t)} U_{\bar{c}_{\sigma(t)}^{*}}^{\prime} f_{x}\left(\bar{a}_{\sigma(t)}^{*}, \bar{a}_{\sigma(t+1)}^{*}, \bar{y}_{\sigma(t+1)}, \bar{r}_{\sigma(t+1)}\right]=0,\right.
\end{array}\right.
$$


where $E_{t}$ represents the conditional expectation on the available information at time $t$, and the information is included in $I_{t}$ which contains the observations on $c_{i, t}, y_{i, t}, r_{i, t}$, and their past values; $f_{x}$ and $f_{y}$ are partial derivatives of function $f$ which is given in NSDM (19) with respect to the first and second variables, respectively.

The transversality condition and Euler equation are often used to describe the optimal paths of dynamic economic models. There are two main explanations for the transversality condition: (1) when the marginal utility is greater than 0 , agents have the possibility of improving welfare near the end of life, and wealth is completely used for consumption. (2) When the marginal utility is equal to 0 , the welfare of the agents cannot be improved. Usually, when the end point is fixed, there are several paths satisfying the Euler equation. The purpose of the transversality condition is to select the optimal path from the paths satisfying the Euler equation.

The transversality condition for NSDM (19) is given by

$$
\left\{\begin{array}{l}
\lim _{T \longrightarrow \infty} E_{0}\left[e_{\left(\beta_{i}-1\right) / \mu}(T, 0) \mu(T) U_{c_{i, T}^{*}}^{\prime} f_{y}\left(a_{i, T}^{*}, a_{i, \sigma(T)}^{*}, y_{i, \sigma(T)}, r_{i, \sigma(T)}\right) a_{i, \sigma(T)}^{*}\right]=0, \\
\lim _{T \longrightarrow \infty} E_{0}\left[e_{\left(\beta_{i}-1\right) / \mu}(T, 0) \mu(T) U_{\bar{a}_{T}^{*}}^{\prime} f_{y}\left(\bar{a}_{T}^{*}, \bar{a}_{\sigma(T)}^{*}, \bar{y}_{\sigma(T)}, \bar{r}_{\sigma(T)}\right) \bar{a}_{\sigma(T)}^{*}\right]=0 .
\end{array}\right.
$$

From Euler equation (22) and transversality condition (23), the optimal solution of NSDM (19) is found. In other words, Euler equation (22) and transversality condition (23) are sufficient for the existence of the optimal solution of NSDM (19).

Theorem 1. If sequence $\left\{c_{i, t}^{*}, a_{i, t}^{*}\right\}_{t=0}^{\infty}$ meets systems (22) and (23), then it is the optimal sequence for NSDM (19).
Proof. We assume that sequence $\left\{c_{i, t}^{*}, a_{i, t}^{*}\right\}_{t=0}^{\infty}$ meets systems (22) and (23). To prove Theorem 1, it is sufficient to prove that the difference between the values of the objective function of NSDM (19) at $\left\{c_{i, t}^{*}, a_{i, t}^{*}\right\}_{t=0}^{\infty}$ and $\left\{c_{i, t}, a_{i, t}\right\}_{t=0}^{\infty}$ is nonnegative, namely,

$$
(\mathrm{NSDM})^{*}-(\mathrm{NSDM})=E_{0} \lim _{T \rightarrow \infty}\left\{\sum_{t \in[0, \sigma(T)) \pi} e_{\left(\beta_{i}-1\right) / \mu}(t, 0) \mu_{t}\left[U\left(c_{i, t}^{*}, \bar{c}_{t}^{*}\right)-U\left(c_{i, t}, \bar{c}_{t}\right)\right]\right\} \geq 0
$$

where $(\mathrm{NSDM})^{*}$ and (NSDM) are the values of the objective function of NSDM (19) at $\left\{c_{i, t}^{*}, a_{i, t}^{*}\right\}_{t=0}^{\infty}$ and $\left\{c_{i, t}, a_{i, t}\right\}_{t=0}^{\infty}$, respectively.
Using the Dominated Convergence Theorem [33], we get

$$
\begin{aligned}
(\mathrm{NSDM})^{*}-(\mathrm{NSDM}) & =\lim _{T \longrightarrow \infty} E_{0}\left\{\sum_{t \in[0, \sigma(T)) \pi} e_{\left(\beta_{i}-1\right) / \mu}(t, 0) \mu_{t}\left[U\left(c_{i, t}^{*}, \bar{c}_{t}^{*}\right)-U\left(c_{i, t}, \bar{c}_{t}\right)\right]\right\} \\
& \geq \lim _{T \longrightarrow \infty} E_{0}\left\{\sum_{t \in[0, \sigma(T)) \pi} e_{\left(\beta_{i}-1\right) / \mu}(t, 0) \mu_{t}\left[U_{c_{i, t}^{*}}^{\prime}\left(c_{i, t}^{*}-c_{i, t}\right)+U_{\bar{c}_{t}^{*}}\left(\bar{c}_{t}^{*}-\bar{c}_{t}\right)\right]\right\} .
\end{aligned}
$$

From equations (20) and (21), we obtain

$$
\begin{aligned}
c_{i, t}^{*} & -c_{i, t} \geq f_{x}\left(a_{i, t}^{*}, a_{i, \sigma(t)}^{*}, y_{i, \sigma(t)}, r_{i, \sigma(t)}\right)\left(a_{i, t}^{*}-a_{i, t}\right) \\
& +f_{y}\left(a_{i, t}^{*}, a_{i, \sigma(t)}^{*}, y_{i, \sigma(t)}, r_{i, \sigma(t)}\right)\left(a_{i, \sigma(t)}^{*}-a_{i, \sigma(t)}\right),
\end{aligned}
$$

$$
\begin{aligned}
\bar{c}_{t}^{*} & -\bar{c}_{t} \geq f_{x}\left(\bar{a}_{t}^{*}, \bar{a}_{\sigma(t)}^{*}, \bar{y}_{\sigma(t)}, \bar{r}_{\sigma(t)}\right)\left(\bar{a}_{t}^{*}-\bar{a}_{t}\right) \\
& +f_{y}\left(\bar{a}_{t}^{*}, \bar{a}_{\sigma(t)}^{*}, \bar{y}_{\sigma(t)}, \bar{r}_{\sigma(t)}\right)\left(\bar{a}_{\sigma(t)}^{*}-\bar{a}_{\sigma(t)}\right) .
\end{aligned}
$$

Substituting equations (26) and (27) into inequality (25), we have 


$$
\begin{aligned}
(\mathrm{NSDM})^{*}-(\mathrm{NSDM}) \geq & \lim _{T \longrightarrow \infty} E_{0} \sum_{t \in[0, \sigma(T)) \mathbb{T}} e_{\left(\beta_{i}-1\right) / \mu}(t, 0) \mu_{t} U_{c_{i, t}^{*}}^{\prime}\left\{f_{x}\left(a_{i, t}^{*}, a_{i, \sigma(t)}^{*}, y_{i, \sigma(t)}, r_{i, \sigma(t)}\right)\left(a_{i, t}^{*}-a_{i, t}\right)\right. \\
& \left.+f_{y}\left(a_{i, t}^{*}, a_{i, \sigma(t)}^{*}, y_{i, \sigma(t)}, r_{i, \sigma(t)}\right)\left(a_{i, \sigma(t)}^{*}-a_{i, \sigma(t)}\right)\right\}+\lim _{T \longrightarrow \infty} E_{0} \sum_{t \in[0, \sigma(T)) \mathbb{T}} e_{\left(\beta_{i}-1\right) / \mu}(t, 0) \mu_{t} U_{\bar{c}_{t}^{*}}^{\prime} \\
& \cdot\left\{f_{x}\left(\bar{a}_{t}^{*}, \bar{a}_{\sigma(t)}^{*}, \bar{y}_{\sigma(t)}, \bar{r}_{\sigma(t)}\right) \times\left(\bar{a}_{t}^{*}-\bar{a}_{t}\right)+f_{y}\left(\bar{a}_{t}^{*}, \bar{a}_{\sigma(t)}^{*}, \bar{y}_{\sigma(t)}, \bar{r}_{\sigma(t)}\right)\left(\bar{a}_{\sigma(t)}^{*}-\bar{a}_{\sigma(t)}\right)\right\}=I .
\end{aligned}
$$

Utilizing the concavity assumption on the function $f(x, y, z, u)$, we obtain

$$
\begin{aligned}
I= & \lim _{T \longrightarrow \infty} E_{0}\left\{\left[e_{\left(\beta_{i}-1\right) / \mu}(0,0) \mu_{0} U_{c_{i, 0}^{*}}^{\prime} f_{x}\left(a_{i, 0}^{*}, a_{i, \sigma(0)}^{*}, y_{i, \sigma(0)}, r_{i, \sigma(0)}\right)\right.\right. \\
& \times\left(a_{i, 0}^{*}-a_{i, 0}\right)+e_{\left(\beta_{i}-1\right) / \mu}(0,0) \mu_{0} U_{\bar{c}_{0}^{*}}^{\prime} f_{x}\left(\bar{a}_{0}^{*}, \bar{a}_{\sigma(0)}^{*}, \bar{y}_{\sigma(0)}, \bar{r}_{\sigma(0)}\right) \\
& \times\left(\bar{a}_{0}^{*}-\bar{a}_{0}\right)+e_{\left(\beta_{i}-1\right) / \mu}(\sigma(0), 0) \mu_{\sigma(0)} U_{c_{i, \sigma(0)}^{*}}^{\prime} f_{x}\left(a_{i, \sigma(0)}^{*}, a_{i, \sigma(1)}^{*}, y_{i, \sigma(1)}, r_{i, \sigma(1)}\right) \\
& \times\left(a_{i, \sigma(0)}^{*}-a_{i, \sigma(0)}\right)+e_{\left(\beta_{i}-1\right) / \mu}(\sigma(0), 0) \mu_{\sigma(0)} U_{\bar{c}_{\sigma(0)}^{*}}^{\prime} \\
& \times f_{x}\left(\bar{a}_{\sigma(0)}^{*}, \bar{a}_{\sigma(1)}^{*}, \bar{y}_{\sigma(1)}, \bar{r}_{\sigma(1)}\right)\left(\bar{a}_{\sigma(0)}^{*}-\bar{a}_{\sigma(0)}\right)+\cdots \\
& +e_{\left(\beta_{i}-1\right) / \mu}(T, 0) \mu_{T} U_{c_{i, T}^{*}}^{\prime} f_{x}\left(a_{i, T}^{*}, a_{i, \sigma(T)}^{*}, y_{i, \sigma(T)}, r_{i, \sigma(T)}\right)\left(a_{i, T}^{*}-a_{i, T}\right) \\
& \left.\left.+e_{\left(\beta_{i}-1\right) / \mu}(T, 0) \mu_{T} U_{\bar{c}_{t}^{*}}^{\prime} f_{x}\left(\bar{a}_{T}^{*}, \bar{a}_{\sigma(T)}^{*}, \bar{y}_{\sigma(T)}, \bar{r}_{\sigma(T)}\right)\left(\bar{a}_{T}^{*}-\bar{a}_{T}\right)\right]\right\} \\
& +\lim _{T \longrightarrow \infty} E_{0} \sum_{t \in[0, \sigma(T)) \pi} e_{\left(\beta_{i}-1\right) / \mu}(t, 0) \mu_{t}\left\{U_{c_{i, t}^{*}}^{\prime} f_{y}\left(a_{i, t}^{*}, a_{i, \sigma(t)}^{*}, y_{i, \sigma(t)}, r_{i, \sigma(t)}\right)\right. \\
& \left.\times\left(a_{i, \sigma(t)}^{*}-a_{i, \sigma(t)}\right)+U_{\bar{c}_{t}^{*}}^{\prime} f_{y}\left(\bar{a}_{t}^{*}, \bar{a}_{\sigma(t)}^{*}, \bar{y}_{\sigma(t)}, \bar{r}_{\sigma(t)}\right)\left(\bar{a}_{\sigma(t)}^{*}-\bar{a}_{\sigma(t)}\right)\right\} .
\end{aligned}
$$

Since $a_{i, 0}=a_{i, 0}^{*}$ and $\bar{a}_{0}=\bar{a}_{*}^{0}$ at the initial state, equation (29) becomes

$$
\begin{aligned}
I= & \lim _{T \longrightarrow \infty}\left[e_{\left(\beta_{i}-1\right) / \mu}(\sigma(0), 0) \mu_{\sigma(0)} U_{c_{i, \sigma(0)}^{*}}^{\prime} f_{x}\left(a_{i, \sigma(0)}^{*}, a_{i, \sigma(1)}^{*}, y_{i, \sigma(1)}, r_{i, \sigma(1)}\right)\right. \\
& \times\left(a_{i, \sigma(0)}^{*}-a_{i, \sigma(0)}\right)+e_{\left(\beta_{i}-1\right) / \mu}(\sigma(0), 0) \mu_{\sigma(0)} U_{\bar{c}_{\sigma(0)}^{*}}^{\prime} \\
& \times f_{x}\left(\bar{a}_{\sigma(0)}^{*}, \bar{a}_{\sigma(1)}^{*}, \bar{y}_{i, \sigma(1)}, \bar{r}_{i, \sigma(1)}\right)\left(\bar{a}_{\sigma(0)}^{*}-\bar{a}_{\sigma(0)}\right)+\cdots \\
& +e_{\left(\beta_{i}-1\right) / \mu}(T, 0) \mu_{T} U_{c_{i, T}^{*}}^{\prime} f_{x}\left(a_{i, T}^{*}, a_{i, \sigma(T)}^{*}, y_{i, \sigma(T)}, r_{i, \sigma(T)}\right)\left(a_{i, T}^{*}-a_{i, T}\right) \\
& \left.+e_{\left(\beta_{i}-1\right) / \mu}(T, 0) \mu_{T} U_{\bar{c}_{T}^{*}}^{\prime} f_{x}\left(\bar{a}_{T}^{*}, \bar{a}_{\sigma(T)}^{*}, \bar{y}_{i, \sigma(T)}, \bar{r}_{i, \sigma(T)}\right)\left(\bar{a}_{T}^{*}-\bar{a}_{T}\right)\right] \\
& +\lim _{T \longrightarrow \infty} E_{0} \sum_{t \in[0, \sigma(T)) \mathbb{T}} e_{\left(\beta_{i}-1\right) / \mu}(t, 0) \mu_{t}\left\{U_{c_{i, t}^{*}}^{\prime} f_{y}\left(a_{i, t}^{*}, a_{i, \sigma(t)}^{*}, y_{i, \sigma(t)}, r_{i, \sigma(t)}\right)\right. \\
& \left.\times\left(a_{i, \sigma(t)}^{*}-a_{i, \sigma(t)}\right)+U_{\bar{c}_{T}^{*}}^{\prime} f_{y}\left(\bar{a}_{t}^{*}, \bar{a}_{\sigma(t)}^{*}, \bar{y}_{i, \sigma(t)}, \bar{r}_{i, \sigma(t)}\right)\left(\bar{a}_{\sigma(t)}^{*}-\bar{a}_{\sigma(t)}\right)\right\} .
\end{aligned}
$$

From the recurrence relation of the exponential function, we obtain $e_{\left(\beta_{i}-1\right) / \mu}(\sigma(t), 0)=\beta_{i} e_{\left(\beta_{i}-1\right) / \mu}(t, 0)$. Consequently, equation (30) is turned into 


$$
\begin{aligned}
I= & \lim _{T \longrightarrow \infty} E_{0} \sum_{t \in[0, T) \mathbb{T}} \beta_{i} e_{\left(\beta_{i}-1\right) / \mu}(t, 0) \mu_{\sigma(t)}\left\{U_{c_{i, \sigma(t)}^{*}}^{\prime} \times f_{x}\left(a_{i, \sigma(t)}^{*}, a_{i, \sigma(t+1)}^{*}, y_{i, \sigma(t+1)}, r_{i, \sigma(t+1)}\right)\left(a_{i, \sigma(t)}^{*}-a_{i, \sigma(t)}\right)\right. \\
& \left.+U_{\bar{c}_{\sigma(t)}^{*}}^{\prime} f_{x}\left(\bar{a}_{\sigma(t)}^{*}, \bar{a}_{\sigma(t+1)}^{*}, \bar{y}_{\sigma(t+1)}, \bar{r}_{\sigma(t+1)}\right)\left(\bar{a}_{\sigma(t)}^{*}-\bar{a}_{\sigma(t)}\right)\right\} \\
& +\lim _{T \longrightarrow \infty} E_{0} \sum_{t \in[0, \sigma(T)) \mathbb{T}} e_{\left(\beta_{i}-1\right) / \mu}(t, 0) \mu_{t}\left\{U_{c_{i, t}^{*}}^{\prime} f_{y}\left(\bar{a}_{t}^{*}, \bar{a}_{\sigma(t)}^{*}, \bar{y}_{\sigma(t)}, \bar{r}_{\sigma(t)}\right) \times\left(\bar{a}_{\sigma(t)}^{*}-\bar{a}_{\sigma(t)}\right)\right. \\
& \left.+U_{\bar{c}_{t}^{*}}^{\prime} f_{y}\left(\bar{a}_{t}^{*}, \bar{a}_{\sigma(t)}^{*}, \bar{y}_{\sigma(t)}, \bar{r}_{\sigma(t)}\right)\left(\bar{a}_{\sigma(t)}^{*}-\bar{a}_{\sigma(t)}\right)\right\}
\end{aligned}
$$

or

$$
\begin{aligned}
I= & \lim _{T \longrightarrow \infty} E_{0} \sum_{t \in[0, T) \mathbb{T}} e_{\left(\beta_{i}-1\right) / \mu}(t, 0)\left\{\beta_{i} \mu_{\sigma(t)} U_{c_{i, \sigma(t)}^{*}}^{\prime} \times f_{x}\left(a_{i, \sigma(t)}^{*}, a_{i, \sigma(t+1)}^{*}, y_{i, \sigma(t+1)}, r_{i, \sigma(t+1)}\right)\left(a_{i, \sigma(t)}^{*}-a_{i, \sigma(t)}\right)\right. \\
& \left.+\mu_{t} U_{c_{i, t}^{*}}^{\prime} f_{y}\left(a_{i, t}^{*}, a_{i, \sigma(t)}^{*}, y_{i, \sigma(t)}, r_{i, \sigma(t)}\right)\left(a_{i, \sigma(t)}^{*}-a_{i, \sigma(t)}\right)\right\} \\
& +\lim _{T \longrightarrow \infty} E_{0} \sum_{t \in[0, T) \mathbb{I}} e_{\left(\beta_{i}-1\right) / \mu}(t, 0)\left\{\beta_{i} \mu_{\sigma(t)} U_{\bar{c}_{\sigma(t)}^{*}}^{\prime} \times f_{x}\left(\bar{a}_{\sigma(t)}^{*}, \bar{a}_{\sigma(t+1)}^{*} \bar{y}_{\sigma(t+1)}, \bar{r}_{\sigma(t+1)}\right)\left(\bar{a}_{\sigma(t)}^{*}-\bar{a}_{\sigma(t)}\right)\right. \\
& \left.+\mu_{t} U_{\bar{c}_{t}^{*}}^{\prime} f_{y}\left(\bar{a}_{\sigma(t)}^{*}, \bar{a}_{\sigma(t+1)}^{*}, \bar{y}_{\sigma(t+1)}, \bar{r}_{\sigma(t+1)}\right)\left(\bar{a}_{\sigma(t)}^{*}-\bar{a}_{\sigma(t)}\right)\right\} \\
& +\lim _{T \longrightarrow \infty} E_{0}\left\{e\left(\beta_{i}-1\right) / \mu\right. \\
& \left.\times f_{y}(T, 0) \mu_{T}^{*} U_{c_{i, T}^{*}}^{\prime} f_{y}\left(\bar{a}_{\sigma(T)}^{*}, \bar{y}_{\sigma(T)}^{*}, a_{i, \sigma(T)}^{*}, y_{i, \sigma(T)}, r_{i, \sigma(T)}\right) \times\left(a_{i, \sigma(T)}^{*}-a_{i, \sigma(T)}\right)+e\left(\bar{a}_{\sigma(T)}^{*}-\bar{a}_{\sigma(T)}\right)\right\} .
\end{aligned}
$$

From system (22), we get

$$
\begin{aligned}
I= & +\lim _{T \longrightarrow \infty} E_{0} e_{\left(\beta_{i}-1\right) / \mu}(T, 0) \mu_{T} \\
& \times\left\{U_{c_{i, T}^{*}}^{\prime} f_{y}\left(a_{i, T}^{*}, a_{i, \sigma(T)}^{*}, y_{i, \sigma(T)}, r_{i, \sigma(T)}\right)\left(a_{i, \sigma(T)}^{*}-a_{i, \sigma(T)}\right)+U_{\bar{c}_{T}^{*}}^{\prime} f_{y}\left(\bar{a}_{T}^{*}, \bar{a}_{\sigma(T)}^{*}, \bar{y}_{\sigma(T)}, \bar{r}_{\sigma(T)}\right)\left(\bar{a}_{\sigma(T)}^{*}-\bar{a}_{\sigma(T)}\right)\right\} .
\end{aligned}
$$

Employing system (23) and $f_{y} \leq 0$, we obtain

$$
\begin{aligned}
I \geq & \lim _{T \longrightarrow \infty} E_{0} e_{\left(\beta_{i}-1\right) / \mu}(T, 0) \mu_{T} \\
& \times\left\{U_{c_{i, T}^{*}}^{\prime} f_{y}\left(a_{i, T}^{*}, a_{i, \sigma(T)}^{*}, y_{i, \sigma(T)}, r_{i, \sigma(T)}\right)\left(a_{i, \sigma(T)}^{*}-a_{i, \sigma(T)}\right)+U_{\bar{c}_{T}^{*}}^{\prime} f_{y}\left(\bar{a}_{T}^{*}, \bar{a}_{\sigma(T)}^{*}, \bar{y}_{\sigma(T)}, \bar{r}_{\sigma(T)}\right)\left(\bar{a}_{\sigma(T)}^{*}-\bar{a}_{\sigma(T)}\right)\right\}=0 .
\end{aligned}
$$

Thus, $(\mathrm{NSDM})^{*}-(\mathrm{NSDM}) \geq 0$. This proof is completed.

\section{The Wealth Distribution Model with the HARA Utility Function}

Inspired by the works in $[17,21]$, the wealth distribution model with the HARA utility function is given by 


$$
\begin{array}{ll}
\max _{\left\{c_{i, t}, a_{i, t}\right\}_{t=0}^{\infty}} & E_{0} \sum_{t=0}^{\infty} \beta_{i}^{t} \frac{\left[c_{i, t}-\gamma_{i} \bar{c}_{t}\right]^{1-\delta_{i}}-1}{1-\delta_{i}} \\
\text { s.t. } & \left\{\begin{array}{l}
a_{i, t}=\left(1+r_{t}\right) \lambda\left(a_{i, t-1}-c_{i, t-1}\right)-(1-\lambda)\left(a_{i, t-1}-c_{i, t-1}\right)+y_{i, t}, \\
\bar{a}_{t}=\left(1+r_{t}\right) \lambda\left(\bar{a}_{t-1}-\bar{c}_{t-1}\right)-(1-\lambda)\left(\bar{a}_{t-1}-\bar{c}_{t-1}\right)+\bar{y}_{t}, \\
\log y_{i, t}=\left(1-\phi_{i}\right) \log y_{i, 0}+\phi_{i} \log y_{i, t-1}+\varepsilon_{i, t}, \\
\log r_{t}=\varphi \log r_{0}+(1-\varphi) \log r_{t-1}+\eta_{t}, \\
\varepsilon_{i, t}, \bar{\varepsilon}_{t}, \eta_{t}, \text { i.i.d. } N\left(0 ; \sigma^{2}\right), \\
a_{i, 0}, \bar{a}_{0}, c_{i, 0}, \bar{c}_{0}, y_{i, 0}, \bar{y}_{0}, r_{0}, \quad \operatorname{are~given,~}
\end{array}\right.
\end{array}
$$

where $E_{0}$ is the conditional expectation, $c_{i, t}(i=1,2, \ldots, n)$ denotes the consumption of agent $i$ at time $t, \bar{c}_{t}$ represents the average consumption of agents in the economy at time $t$, $0<\varphi<1,0<\bar{\phi}<1,0<\phi_{i}<1,0<\gamma_{i}<1 \quad(i=1,2, \ldots, n)$, all agents' consumption in the economy satisfies $c_{i, t}>\gamma_{i} \bar{c}_{t}$ by limiting the value of $\gamma_{i}, 0<\lambda<1$ is the saving rate of agent $i$, $a_{i, t}(i=1,2, \ldots, n)$ denotes the wealth of agent $i$ at time $t, \bar{a}_{t}$ is the average wealth of agents at time $t, y_{i, t}(i=1,2, \ldots, n)$ represents the earnings of agent $i$ at time $t, \bar{y}_{t}$ is the average income of agents at time $t, r_{t}$ denotes the rate of return of agents on wealth process at time $t$, all agents are assumed to have the same rate of return at the same time, and the discount factor $0<\beta_{i}<1 \quad(i=1,2, \ldots, n)$ and the relative risk aversion parameter $0<\delta_{i}<1 \quad(i=1,2, \ldots, n)$ are different to each agent $i$ and evolve stochastically.

In model (34), the wealth of agent $i$ in time $t$ is divided into two parts. Part of the wealth comes from the income that the agent uses part of the remaining wealth in time $t-1$ for savings, that is, $\left(1+r_{t}\right) \lambda\left(a_{i, t-1}-c_{i, t-1}\right)$, where $a_{i, t-1}-$ $c_{i, t-1}$ is the remaining wealth of the previous. The second part of the wealth comes from market transaction, namely, $y_{i, t}-(1-\lambda)\left(a_{i, t-1}-c_{i, t-1}\right)$, where $y_{i, t}$ is the wealth obtained by agent $i$ in the transaction, and $(1-\lambda)\left(a_{i, t-1}-c_{i, t-1}\right)$ indicates that agent $i$ uses part of the remaining wealth in time $t-1$ for trading.

In model (34), agents are allowed to use part of the remaining wealth at time $t-1$ for trading and part for savings. Agents adopt specific trading strategies that determine whether the agent gains or loses in wealth exchange to maximize their utility. In model $(34), y_{i, t}-(1-\lambda)\left(a_{i, t-1}-\right.$ $\left.c_{i, t-1}\right)>0(i=1,2, \ldots, n)$ denotes the net profits of agent $i$ from wealth exchange, and $y_{i, t}-(1-\lambda)\left(a_{i, t-1}-c_{i, t-1}\right)<0$ $(i=1,2, \ldots, n)$ represents the amount of wealth lost by agent $i$ in the process of wealth exchange. When $y_{i, t}-(1-$ d) $\left(a_{i, t-1}-c_{i, t-1}\right)=0(i=1,2, \ldots, n)$ in model (34), agent $i$ does not make profits in the process of wealth exchange.
The utility function of model (34) is

$$
U\left(c_{i, t}, \bar{c}_{t}\right)=\frac{\left[c_{i, t}-\gamma_{i} \bar{c}_{t}\right]^{1-\delta_{i}}-1}{1-\delta_{i}},
$$

which describes the preference of agent $i$ for risk. Equation (35) is so-called the HARA utility function which captures the idea of "keeping up with the Joneses," namely, the consumption standards of agents are affected by average consumption level. When time $t$ is fixed, $\bar{c}_{t}$ becomes a constant parameter $\bar{c}$ which plays an important role in determining agent's preferences.

(i) When $\bar{c}>0$, utility function (35) describes an agent with decreasing relative risk aversion (DRRA) preference, and $\bar{c}$ is interpreted as the minimum standard of living for consumption. In utility function (35), when $c_{i, t} \longrightarrow \bar{c}$, the marginal utility tends to infinity, and the optimal consumption level will never reach $\bar{c}_{t}=\bar{c}$ and always satisfies $c_{i, t}>\bar{c}$ (see Achury et al. [26]).

(ii) When $\bar{c}<0$, utility function (35) characterizes an agent with increasing relative risk aversion (IRRA) preference. $\bar{c}$ in utility function (35) indicates that agents become more conservative as their consumption increases. However, $\bar{c}$ is no longer interpreted as the agent's minimum consumption level. Levaggi and Menoncin [27] introduce the increasing relative risk aversion preference.

(iii) When $\bar{c}=0$, utility function (35) describes an agent with constant relative risk aversion preference which is the CRRA utility function.

We set the isolated discrete time domains $\mathbb{T}$ to satisfy sup $\mathbb{T}=\infty$. Let $\mathbb{T} \cap[0, \infty)=[0, \infty)$. From model $(34)$, the wealth distribution model on isolated discrete time domains $\mathbb{T}$ has the following form: 


$$
\begin{aligned}
& \sup _{\left\{c_{i, t}, a_{i, t}\right\}_{t=0}^{\infty}} E_{0} \sum_{t \in[0, \infty) \pi}\left[e_{\left(\beta_{i}-1\right) / \mu}(t, 0) \frac{\left[c_{i, t}-\gamma_{i} \bar{c}_{t}\right]^{1-\delta_{i}}-1}{1-\delta_{i}} \mu_{t}\right], \\
& \text { s.t. }\left\{\begin{array}{l}
c_{i, t}=a_{i, t}-\frac{a_{i, \sigma(t)}}{2 \lambda+\lambda r_{\sigma(t)}-1}+\frac{y_{i, \sigma(t)}}{2 \lambda+\lambda r_{\sigma(t)}-1}, \\
\bar{c}_{t}=\bar{a}_{t}-\frac{\bar{a}_{\sigma(t)}}{2 \lambda+\lambda r_{\sigma(t)}-1}+\frac{\bar{y}_{\sigma(t)}}{2 \lambda+\lambda r_{\sigma(t)}-1}, \\
\log y_{i, t}=\left(1-\phi_{i}\right) \log y_{i, 0}+\phi_{i} \log y_{i, p(t)}+\varepsilon_{i, t}, \\
\log \bar{y}_{t}=(1-\bar{\phi}) \log \bar{y}_{0}+\bar{\phi} \log \bar{y}_{p(t)}+\bar{\varepsilon}_{t}, \\
\log r_{t}=\varphi \log r_{0}+(1-\varphi) \log r_{p(t)}+\eta_{t}, \\
\varepsilon_{i, t}, \bar{\varepsilon}_{t}, \eta_{t} \sim \text { i.i.d. } N\left(0, \sigma^{2}\right), \\
a_{i, 0}, a_{i, \sigma(0)}, c_{i, 0}, \bar{c}_{0}, y_{i, 0}, \bar{y}_{0}, r_{0} \quad \text { are given, }
\end{array}\right.
\end{aligned}
$$

where $\delta_{i} \neq 1,2 \lambda+\lambda r_{\sigma(t)}-1 \neq 0(i=1,2, \ldots, n), \sigma: \mathbb{T} \longrightarrow \mathbb{T}$ is the forward jump operator, and $a_{i, t}$ and $a_{i, \sigma(t)}$ are values of $a_{i}$ on two consecutive points on the isolated discrete time domains. $p: \mathbb{T} \longrightarrow \mathbb{T}$ is the backward jump operator.

Remark 1. When $\mathbb{T}=\mathbb{Z}$, wealth distribution model (36) becomes model (34). Consequently, model (36) is a generalization of model (34).
Remark 2. When $\mathbb{T}=\mathbb{Z}, \lambda=1$, and $y_{i, t}=0$, i.e., wealth exchange is not considered, model (36) is rewritten as

$$
\begin{array}{ll}
\max _{\left\{c_{i, t}, a_{i, t}\right\}_{t=0}^{\infty}} & E_{0} \sum_{t=0}^{\infty} \beta_{i}^{\beta_{c}} \frac{\mathcal{c}_{i, t}^{1-\sigma_{i}}-1}{1-\sigma_{i}} \\
\text { s.t. } & \left\{\begin{array}{l}
a_{i, t}=\left(1+r_{t}\right) \lambda\left(a_{i, t-1}-c_{i, t-1}\right) \\
c_{i, t-1}<a_{i, t-1},
\end{array}\right.
\end{array}
$$

where $1+r_{t}$ is equivalent to the agent's idiosyncratic rate of return on wealth process. This model is consistent with that in [10], and the stationary distribution for $a_{i, t}$ which satisfies the wealth accumulation equation in model (34) has a fat tail. This conclusion is proved by Benhabib et al. in [10].

To analyze the distribution of wealth among agents, the method in [21] is employed to find the optimal solution of model (36). The steps to find the optimal solution of model (36) are as follows: (I) constructing the Euler equation and transversality condition for model (36); (II) finding the initial state of the constraint conditions in model (36); (III) log-linearizing the constraints and the Euler equation in Step I; (IV) expressing the corresponding recursive equations by matrix operators; and (V) finding the optimal solution of model (36) by using the method of solving the rational expectation model on isolated discrete time domains.

Step I. Construction of the Euler equation and transversality condition.

From system (37), the Euler equation of model (36) is given by

$$
\left\{\begin{array}{l}
E_{t}\left[\mu_{t}\left(c_{i, t}-\gamma_{i} \bar{c}_{t}\right)^{-\delta_{i}}\left(-\frac{1}{2 \lambda+\lambda r_{\sigma(t)}-1}\right)+\beta_{i} \mu_{\sigma(t)}\left(c_{i, \sigma(t)}-\gamma_{i} \bar{c}_{\sigma(t)}\right)^{-\delta_{i}}\right]=0, \\
E_{t}\left[\mu_{t}\left(c_{i, t}-\bar{\gamma} \bar{c}_{t}\right)^{-\delta}\left(\frac{\bar{\gamma}}{2 \lambda+\lambda r_{\sigma(t)}-1}\right)-\beta \mu_{\sigma(t)}\left(c_{i, \sigma(t)}-\bar{\gamma} \bar{c}_{\sigma(t)}\right)^{-\delta} \bar{\gamma}\right]=0,
\end{array}\right.
$$

where $\delta$ is a relative risk aversion parameter in the mean sense, $\beta$ is the average discount factor, and $\bar{\gamma}$ is the average of $\gamma_{i}(i=1,2, \ldots, n)$.

Consequently, the first-order condition of model (36) is

$$
\left\{\begin{array}{l}
E_{t}\left[\beta \frac{\mu_{\sigma(t)}}{\mu_{t}}\left(\frac{c_{i, t}-\gamma_{i} \bar{c}_{t}}{c_{i, \sigma(t)}-\gamma_{i} \bar{c}_{\sigma(t)}}\right)^{\delta_{i}} k_{\sigma(t)}\right]=1, \\
E_{t}\left[\beta \frac{\mu_{\sigma(t)}}{\mu_{t}}\left(\frac{c_{i, t}-\bar{\gamma} \bar{c}_{t}}{c_{i, \sigma(t)}-\bar{\gamma} \bar{c}_{\sigma(t)}}\right)^{\delta} k_{\sigma(t)}\right]=1,
\end{array}\right.
$$

where $k_{t}=2 \lambda+\lambda r_{t}-1, c_{i, \sigma(t)}-\gamma_{i} \bar{c}_{\sigma(t)} \neq 0, c_{i, \sigma(t)}-\overline{\gamma c}_{\sigma(t)} \neq 0$ $(i=1,2, \ldots, n)$, and $\mu_{t} \neq 0$.
Employing system (38), the transversality condition of model (36) becomes

$$
\left\{\begin{array}{l}
\lim _{T \longrightarrow \infty} E_{0}\left[e_{\left(\beta_{i}-1\right) / \mu}(T, 0) \mu_{T}\left(c_{i, T}-\gamma_{i} \bar{c}_{T}\right)^{-\delta_{i}}\left(-\frac{1}{k_{\sigma(T)}}\right) a_{i, \sigma(T)}\right]=0, \\
\lim _{T \longrightarrow \infty} E_{0}\left[e_{\left(\beta_{i}-1\right) / \mu}(T, 0) \mu_{T}\left(c_{i, T}-\bar{\gamma} \bar{c}_{T}\right)^{-\delta}\left(\frac{\bar{\gamma}}{k_{\sigma}(t)}\right) \bar{a}_{\sigma(T)}\right]=0 .
\end{array}\right.
$$

Step II. Expression of the initial state.

To seek out the initial state, the constraints of model (36) are rewritten as follows: 


$$
c_{i, t}=a_{i, t}-\frac{1}{2 \lambda+\lambda r_{\sigma(t)}-1} a_{i, \sigma(t)}+\frac{1}{2 \lambda+\lambda r_{\sigma(t)}-1} y_{i, \sigma(t)}
$$$$
\bar{c}_{t}=\bar{a}_{t}-\frac{1}{2 \lambda+\lambda r_{\sigma(t)}-1} \bar{a}_{\sigma(t)}+\frac{1}{2 \lambda+\lambda r_{\sigma(t)}-1} \bar{y}_{\sigma(t)},
$$

$$
k_{t}=2 \lambda+\lambda r_{t}-1
$$

$$
\log y_{i, t}=\left(1-\phi_{i}\right) \log y_{i, 0}+\phi_{i} \log y_{i, p(t)}+\varepsilon_{i, t},
$$

$$
\log \bar{y}_{t}=(1-\bar{\phi}) \log \bar{y}_{0}+\bar{\phi} \log \bar{y}_{p(t)}+\bar{\varepsilon}_{t}
$$

$$
\log r_{t}=\varphi \log r_{0}+(1-\varphi) \log r_{p(t)}+\eta_{t}
$$

From system (39) and equations (41a)-(41f), the initial states of model (36) are

$$
\begin{gathered}
c_{i, 0}=a_{i, 0}-\frac{1}{2 \lambda+\lambda r_{\sigma(0)}-1} a_{i, \sigma(0)}+\frac{1}{2 \lambda+\lambda r_{\sigma(0)}-1} y_{i, \sigma(0)}, \\
\bar{c}_{0}=\bar{a}_{0}-\frac{1}{2 \lambda+\lambda r_{\sigma(0)}-1} \bar{a}_{\sigma(0)}+\frac{1}{2 \lambda+\lambda r_{\sigma(0)}-1} \bar{y}_{\sigma(0)}, \\
E_{0}\left[\beta_{i} \frac{\mu_{\sigma(0)}}{\mu_{0}}\left(\frac{c_{i, 0}-\gamma_{i} \bar{c}_{0}}{c_{i, \sigma(0)}-\gamma_{i} \bar{c}_{\sigma(0)}}\right)^{\delta_{i}} k_{\sigma(0)}\right]=1 \\
E_{0}\left[\beta \frac{\mu_{\sigma(0)}}{\mu_{0}}\left(\frac{c_{i, 0}-\overline{\gamma c}_{0}}{c_{i, \sigma(0)}-\overline{\gamma c}_{\sigma(0)}}\right)^{\delta} k_{\sigma(0)}\right]=1 \\
\log y_{i, 0}=\left(1-\phi_{i}\right) \log y_{i, 0}+\phi_{i} \log y_{i, p(0)}+\varepsilon_{i, 0} \\
\log \bar{y}_{0}=(1-\bar{\phi}) \log \bar{y}_{0}+\bar{\phi} \log \bar{y}_{p(0)}+\bar{\varepsilon}_{0} \\
\log r_{t}=\varphi \log r_{0}+(1-\varphi) \log r_{p(0)}+\eta_{0}
\end{gathered}
$$

where $2 \lambda+\lambda r_{\sigma(0)}-1 \neq 0(i=1,2, \ldots, n), c_{i, \sigma(0)}-\gamma_{i} \bar{c}_{\sigma(0)} \neq 0$ $(i=1,2, \ldots, n), c_{i, \sigma(0)}-\overline{\gamma c}_{\sigma(0)} \neq 0$, and $\mu_{0} \neq 0$.

Step III. Log-linearization of the constraints and the Euler equation.

Let $\hat{x}_{t}=\left(x_{t}-x_{0}\right) / x_{0}$. To log-linearize equation (41a), our idea is to linearize each part of the equation and then sum it up. For $c_{i, t}$, we have

$$
c_{i, t} \approx c_{i, 0}\left(1+\widehat{c}_{i, t}\right)
$$

Through a series of algebraic calculations, we get (see Appendix A)

$$
\begin{array}{r}
\widehat{c}_{i, t} \approx \frac{a_{i, 0}}{c_{i, 0}} \widehat{a}_{i, t}-\frac{a_{i, \sigma(0)}}{c_{i, 0} k_{\sigma(0)}} \widehat{a}_{i, \sigma(t)}+\frac{y_{i, \sigma(0)}}{c_{i, 0} k_{\sigma(0)}} \widehat{y}_{i, \sigma(t)} \\
+\frac{\lambda r_{\sigma(0)} a_{i, \sigma(0)}}{k_{\sigma(0)}^{2} c_{i, 0}} \widehat{r}_{\sigma(t)}-\frac{\lambda r_{\sigma(0)} y_{i, \sigma(0)}}{k_{\sigma(0)}^{2} c_{i, 0}} \widehat{r}_{\sigma(t)},
\end{array}
$$

where $c_{i, 0} \neq 0(i=1,2, \ldots, n)$ and $k_{\sigma(0)} \neq 0$.

Similarly, we log-linearize equation (41b) and obtain

$$
\begin{aligned}
\widehat{\bar{c}}_{t} \approx & \frac{\bar{a}_{0}}{\bar{c}_{0}} \widehat{\bar{a}}_{t}-\frac{\bar{a}_{\sigma(0)}}{k_{\sigma(0)} \bar{c}_{0}} \widehat{\bar{a}}_{\sigma(t)}+\frac{\bar{y}_{\sigma(0)}}{k_{\sigma(0)} \bar{c}_{0}} \widehat{\bar{y}}_{\sigma(t)} \\
& +\frac{\bar{\lambda}_{\sigma(0)} r_{\sigma(0)} \bar{a}_{\sigma(0)}}{k_{\sigma(0)}^{2} \bar{c}_{0}} \widehat{r}_{\sigma(t)}-\frac{\bar{\lambda}_{\sigma(0)} r_{\sigma(0)} \bar{y}_{\sigma(0)}}{k_{\sigma(0)}^{2} \bar{c}_{0}} \widehat{r}_{\sigma(t)},
\end{aligned}
$$

where $\bar{c}_{0} \neq 0$ and $k_{\sigma(0)} \neq 0$.

We log-linearize two sides of equation (41c) and have

$$
\begin{aligned}
k_{t} & \approx k_{0}\left(1+\widehat{k}_{t}\right) \\
& \approx 2 \lambda+\lambda r_{0}-1+\lambda\left(r_{t}-r_{0}\right) \\
& \approx k_{0}+\lambda r_{0} \widehat{r}_{t} .
\end{aligned}
$$

Dividing two sides of equation (46) by $k_{0}$, we obtain

$$
\widehat{k}_{t}=\frac{\lambda r_{0}}{k_{0}} \widehat{r}_{t}
$$

where $k_{0} \neq 0$.

Log-linearizing system (39), we get (see Appendix B)

$$
\begin{aligned}
& E_{t}\left[\mu_{\sigma(t)}-\mu_{t}+\frac{c_{i, 0} \delta_{i}}{c_{i, 0}-\gamma_{i} \bar{c}_{0}} \widehat{c}_{i, t}-\frac{\delta_{i} \bar{c}_{0} \gamma_{i}}{c_{i, 0}-\gamma_{i} \bar{c}_{0}} \widehat{\bar{c}}_{t}\right. \\
& \left.-\frac{c_{i, \sigma(0)} \delta_{i}}{c_{i, \sigma(0)}-\gamma_{i} \bar{c}_{\sigma(0)}} \widehat{c}_{i, \sigma(t)}-\frac{\delta_{i} \gamma_{i} \bar{c}_{\sigma(0)}}{c_{i, \sigma(0)}-\gamma_{i} \bar{c}_{\sigma(0)}} \widehat{\bar{c}}_{\sigma(t)}+\widehat{k}_{\sigma(t)}\right]=0,
\end{aligned}
$$

$$
\begin{aligned}
& E_{t}\left[\mu_{\sigma(t)}-\mu_{t}+\frac{\delta c_{i, 0}}{c_{i, 0}-\overline{\gamma c}_{0}} \widehat{c}_{i, t}-\frac{\delta \overline{\gamma c}_{0}}{c_{i, 0}-\overline{\gamma c}_{0}} \widehat{\overline{\bar{c}}}_{t}\right. \\
& \left.\quad-\frac{\delta c_{i, \sigma(0)}}{c_{i, \sigma(0)}-\overline{\gamma c}_{\sigma(0)}} \bar{c}_{i, \sigma(t)}+\frac{\delta \overline{\gamma c}}{c_{i, \sigma(0)}-\overline{\gamma c}_{\sigma(0)}} \widehat{\overline{\bar{c}}}_{\sigma(t)}+\widehat{k}_{\sigma(t)}\right]=0 .
\end{aligned}
$$

Log-linearizing equation (41d), we obtain the following expression (see Appendix C):

$$
\widehat{y}_{i, \sigma(t)} \approx \phi_{i} \widehat{y}_{i, t}+\varepsilon_{i, \sigma(t)}-\varepsilon_{i, \sigma(0)},
$$

where $\varepsilon_{i, \sigma(0)}=0$.

Similarly, we log-linearize equation (41e) and get

$$
\widehat{\bar{y}}_{\sigma(t)}=\bar{\phi} \widehat{\bar{y}}_{t}+\bar{\varepsilon}_{\sigma(t)}-\bar{\varepsilon}_{\sigma(0)},
$$

where $\bar{\varepsilon}_{\sigma(0)}=0$.

Taking conditional expectation on both sides of equations (50) and (51) on information set $I_{t}$ yields 


$$
\begin{gathered}
E_{t}\left[\widehat{y}_{i, \sigma(t)}\right] \approx \phi_{i} \hat{y}_{i, t}, \\
E_{t}\left[\hat{\bar{y}}_{\sigma(t)}\right] \approx \bar{\phi} \hat{\bar{y}}_{t} .
\end{gathered}
$$

Log-linearizing equation (41f), we obtain (see Appendix D)

$$
\widehat{r}_{\sigma(t)} \approx(1-\varphi) \widehat{r}_{t}+\eta_{\sigma(t)}-\eta_{\sigma(0)},
$$

where $\eta_{\sigma(0)}=0$.

Adding conditional expectation on both sides of equation (54) on information set $I_{t}$, we have

$$
E_{t}\left[\widehat{r}_{\sigma(t)}\right] \approx(1-\varphi) \widehat{r}_{t}
$$

Step IV. Expressing the corresponding recursive equations by matrix operators.

Taking conditional expectation on both sides of equation (44) on information set $I_{t}$, we get

$$
\begin{aligned}
\frac{a_{i, \sigma(0)}}{c_{i, 0} k_{\sigma(0)}} E_{t}\left[\widehat{a}_{i, \sigma(t)}\right] \approx & \frac{a_{i, 0}}{c_{i, 0}} \widehat{a}_{i, t}-\widehat{c}_{i, t}+\frac{y_{i, \sigma(0)}}{c_{i, 0} k_{\sigma(0)}} E_{t}\left[\widehat{y}_{i, \sigma(t)}\right] \\
& +\frac{\lambda_{i, \sigma(0)} r_{\sigma(0)}}{k_{\sigma(0)}^{2} c_{i, 0}}\left(a_{i, \sigma(0)}-y_{i, \sigma(0)}\right) E_{t}\left[\widehat{r}_{\sigma(t)}\right] .
\end{aligned}
$$

From equations (52) and (55), equation (56) becomes

$$
\begin{gathered}
\frac{a_{i, \sigma(0)}}{c_{i, 0} k_{\sigma(0)}} E_{t}\left[\widehat{a}_{i, \sigma(t)}\right] \approx \frac{a_{i, 0}}{c_{i, 0}} \widehat{a}_{i, t}-\widehat{c}_{i, t}+\frac{y_{i, \sigma(0)}}{c_{i, 0} k_{\sigma(0)}} \phi_{i} \widehat{y}_{i, t} \\
+\frac{\lambda_{i, \sigma(0)} r_{\sigma(0)}}{k_{\sigma(0)}^{2} c_{i, 0}}\left(a_{i, \sigma(0)}-y_{i, \sigma(0)}\right)(1-\varphi) \widehat{r}_{t} .
\end{gathered}
$$

Likewise, equation (45) is rewritten by

$$
\begin{aligned}
\frac{\bar{a}_{\sigma(0)}}{\bar{c}_{0} k_{\sigma(0)}} E_{t}\left[\widehat{\bar{a}}_{\sigma(t)}\right] \approx & \frac{\bar{a}_{0}}{\bar{c}_{0}} \widehat{\bar{a}}_{t}-\widehat{\bar{c}}_{t}+\frac{\bar{y}_{\sigma(0)}}{\bar{c}_{0} k_{\sigma(0)}} \bar{\phi} \widehat{\bar{y}}_{t} \\
& +\frac{\bar{\lambda}_{\sigma(0)} r_{\sigma(0)}}{k_{\sigma(0)}^{2} \bar{c}_{0}}\left(\bar{a}_{\sigma(0)}-\bar{y}_{\sigma(0)}\right)(1-\varphi) \widehat{r}_{t} .
\end{aligned}
$$

From equations (47), (48), and (55), we have

$$
\begin{gathered}
\frac{c_{i, \sigma(0)} \delta_{i}}{c_{i, \sigma(0)}-\gamma_{i} \bar{c}_{\sigma(0)}} E_{t}\left[\widehat{c}_{i, \sigma(t)}\right]-\frac{\delta_{i} \gamma_{i} \bar{c}_{\sigma(0)}}{c_{i, \sigma(0)}-\gamma_{i} \bar{c}_{\sigma(0)}} E_{t}\left[\widehat{\bar{c}}_{\sigma(t)}\right] \\
=\mu_{\sigma(t)}-\mu_{t}+\frac{c_{i, 0} \delta_{i}}{c_{i, 0}-\gamma_{i} \bar{c}_{0}} \widehat{c}_{i, t} \\
-\frac{\delta_{i} \bar{c}_{0} \gamma_{i}}{c_{i, 0}-\gamma_{i} \bar{c}_{0}} \hat{\bar{c}}_{t}+\frac{\lambda_{i, \sigma(0)} r_{\sigma(0)}}{k_{\sigma(0)}}(1-\varphi) \widehat{r}_{t} .
\end{gathered}
$$

Calculating equation (49) gives rise to

$$
\begin{gathered}
\frac{c_{i, \sigma(0)} \delta}{c_{i, \sigma(0)}-\overline{\gamma c}_{\sigma(0)}} E_{t}\left[\widehat{c}_{i, \sigma(t)}\right]-\frac{\delta \overline{\gamma c}_{\sigma(0)}}{c_{i, \sigma(0)}-\overline{\gamma c}_{\sigma(0)}} E_{t}\left[\widehat{\bar{c}}_{\sigma(t)}\right] \\
=\mu_{\sigma(t)}-\mu_{t}+\frac{c_{i, 0} \delta}{c_{i, 0}-\overline{\gamma \mathcal{c}}_{0}} \widehat{c}_{i, t} \\
-\frac{\delta \bar{c}_{0} \bar{\gamma}}{c_{i, 0}-\overline{\gamma \mathcal{c}}_{0}} \widehat{\overline{\bar{c}}}_{t}+\frac{\lambda r_{\sigma(0)}}{k_{\sigma(0)}}(1-\varphi) \widehat{r}_{t} .
\end{gathered}
$$

Equations (52), (53), (55), and (57)-(60) are rewritten by the matrix form, namely,

$$
B E_{t}\left[\begin{array}{c}
\widehat{a}_{i, \sigma(t)} \\
\widehat{\bar{a}}_{\sigma(t)} \\
\widehat{c}_{i, \sigma(t)} \\
\widehat{\bar{c}}_{\sigma(t)} \\
\hat{y}_{i, \sigma(t)} \\
\widehat{\bar{y}}_{\sigma(t)} \\
\widehat{r}_{\sigma(t)}
\end{array}\right]=C\left[\begin{array}{c}
\widehat{a}_{i, t} \\
\widehat{\bar{a}}_{t} \\
\widehat{c}_{i, t} \\
\hat{\bar{c}}_{t} \\
\hat{y}_{i, t} \\
\widehat{\bar{y}}_{t} \\
\widehat{r}_{t}
\end{array}\right]+\left[\begin{array}{c}
0 \\
0 \\
\mu_{\sigma(t)}-\mu_{t} \\
\mu_{\sigma(t)}-\mu_{t} \\
0 \\
0 \\
0
\end{array}\right],
$$


where

\begin{tabular}{|c|c|c|c|c|c|}
\hline$\left[\frac{a_{i, \sigma(0)}}{c_{i, 0} k_{\sigma(0)}}\right.$ & 0 & 0 & 0 & 0 & 00 \\
\hline 0 & $\frac{\bar{a}_{\sigma(0)}}{\bar{c}_{0} k_{\sigma(0)}}$ & 0 & 0 & 0 & 00 \\
\hline 0 & 0 & $c_{i, \sigma(0)} \delta_{i}$ & $c_{i, \sigma(0)} \delta_{i} \gamma_{i}$ & 0 & 00 \\
\hline & & $c_{i, \sigma(0)}-\gamma_{i} \bar{c}_{\sigma(0)}$ & $c_{i, \sigma(0)}-\gamma_{i} \bar{c}_{\sigma(0)}$ & & 8 \\
\hline 0 & 0 & $c_{i, \sigma(0)} \delta$ & $c_{i, \sigma(0)} \delta \bar{\gamma}$ & 0 & 0 \\
\hline & & $c_{i, \sigma(0)}-\overline{\gamma \mathcal{c}}_{\sigma(0)}$ & $c_{i, \sigma(0)}-\overline{\gamma \mathcal{c}}_{\sigma(0)}$ & & \\
\hline 0 & 0 & 0 & 0 & 1 & $\begin{array}{ll}0 & 0\end{array}$ \\
\hline 0 & 0 & 0 & 0 & 0 & 10 \\
\hline 0 & 0 & 0 & 0 & 0 & 0 \\
\hline
\end{tabular}

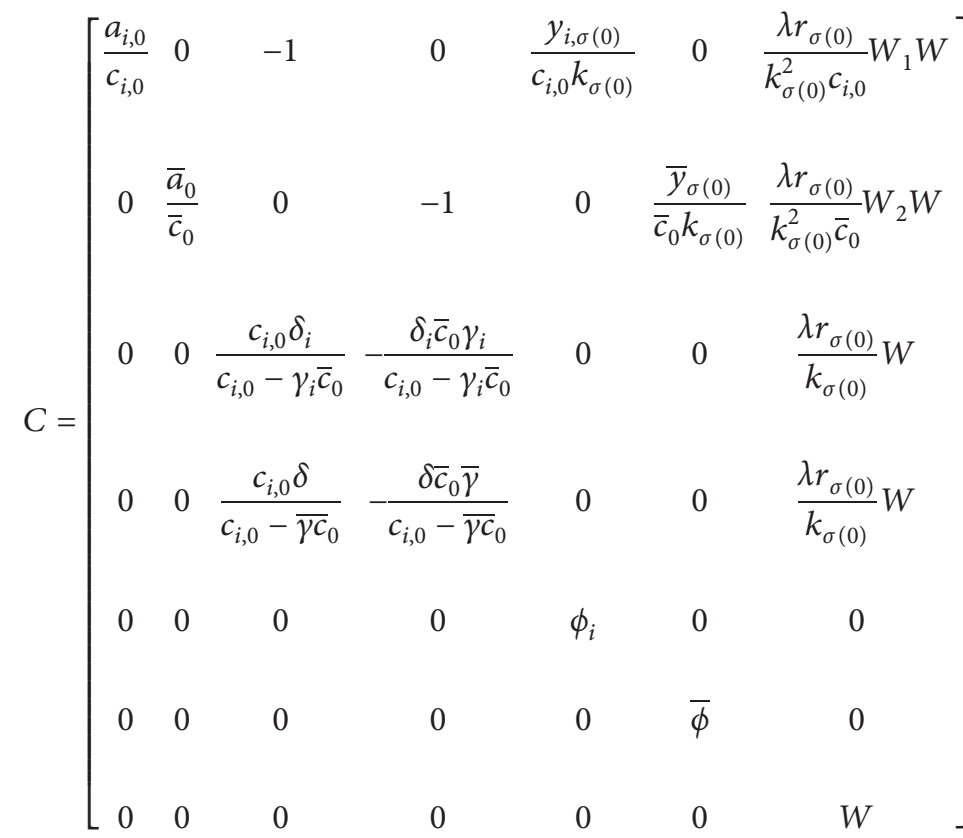

and $c_{i, 0} \neq 0, k_{\sigma(0)} \neq 0, c_{i, \sigma(0)}-\gamma_{i} \bar{c}_{\sigma(0)} \neq 0, c_{i, \sigma(0)}-\overline{\gamma c}_{\sigma(0)} \neq 0$, $c_{i, 0}-\gamma_{i} \bar{c}_{0} \neq 0, c_{i, 0}-\overline{\gamma c}_{0} \neq 0 \quad(i=1,2, \ldots, n), \bar{c}_{0}, W=1-\varphi$, $W_{1}=a_{i, \sigma(0)}-y_{i, \sigma(0)}, \quad W_{2}=\bar{a}_{\sigma(0)}-\bar{y}_{\sigma(0)}$, and $\bar{k}_{\sigma}(0)$ are nonzero constants.

Step $V$. Finding the optimal solution of equation (61).

$$
J_{t}=e_{(I-D) D^{-1}(1 / \mu)}(t, 0) M(t)
$$$$
-e_{(I-D) D^{-1}(1 / \mu)}(t, 0) \int_{0}^{T} e_{\Theta(I-D) D^{-1}(1 / \mu)}(s, 0) \frac{1}{\mu(s)} F(s) \Delta s,
$$

where

Theorem 2. Let $\mathbb{T}$ be the isolated discrete time domains. If matrix $C$ is nonsingular, then the optimal solution of equation (61) is 


$$
\begin{aligned}
J_{t} & =\left[\begin{array}{c}
\hat{a}_{i, t} \\
\hat{\bar{a}}_{t} \\
\widehat{c}_{i, t} \\
\hat{\bar{c}}_{t} \\
\hat{y}_{i, t} \\
\hat{\bar{y}}_{t} \\
\hat{r}_{t}
\end{array}\right], \\
D & =C^{-1} B,
\end{aligned}
$$

$$
F(t)=C^{-1}\left[\begin{array}{c}
0 \\
0 \\
\mu_{\sigma(t)}-\mu_{t} \\
\mu_{\sigma(t)}-\mu_{t} \\
0 \\
0 \\
0
\end{array}\right],
$$

and $M(t)$ is a $7 \times 1$ arbitrary matrix martingale on $\mathbb{T}$ and satisfies the martingale property $E_{t}\left[M^{\sigma}(t)\right]=M(t)$.
Here, we state that the proof of Theorem 2 is similar to that of Lemma 2.3 in [21]. We omit its proof.

Remark 3. If $\mathbb{T}=\mathbb{Z}$, then solution (63) becomes

$$
J_{t}=D^{-t} M(t)-D^{-t} \sum_{s=0}^{t-1} D^{s} F_{s} .
$$

We mention that equation (65) is the optimal solution of model (34).

Remark 4. When $\bar{c}_{t}=\bar{c}, \bar{c}_{t}$ indicates the average consumption level of agents during the whole trading period, and equation (61) becomes

$$
B_{1} E_{t}\left[\begin{array}{c}
\hat{a}_{i, \sigma(t)} \\
\widehat{c}_{i, \sigma(t)} \\
\hat{y}_{i, \sigma(t)} \\
\widehat{r}_{\sigma(t)}
\end{array}\right]=C_{1}\left[\begin{array}{c}
\hat{a}_{i, t} \\
\widehat{c}_{i, t} \\
\hat{y}_{i, t} \\
\hat{r}_{t}
\end{array}\right]+\left[\begin{array}{c}
0 \\
\mu_{\sigma(t)}-\mu_{t} \\
0 \\
0
\end{array}\right],
$$

where

$$
\begin{aligned}
C_{1} & =\left[\begin{array}{cccc}
\frac{a_{i, 0}}{c_{i, 0}} & -1 & \frac{y_{i, \sigma(0)}}{c_{i, 0} k_{\sigma(0)}} \frac{\lambda r_{\sigma(0)}}{k_{\sigma(0)}^{2} c_{i, 0}}\left(a_{i, \sigma(0)}-y_{i, \sigma(0)}\right)(1-\varphi) \\
0 \frac{\delta_{i} c_{i, \sigma(0)}}{c_{i, \sigma(0)}-\gamma_{i} \bar{c}} & 0 & \frac{\lambda r_{\sigma(0)}}{k_{\sigma(0)}}(1-\varphi) \\
0 & 0 & \phi_{i} & 0 \\
0 & 0 & 0 & 1-\varphi
\end{array}\right], \\
B_{1} & =\left[\begin{array}{cccc}
\frac{a_{i, \sigma(0)}}{c_{i, 0} k_{\sigma(0)}} & 0 & 0 & 0 \\
0 & \frac{\delta_{i} c_{i, \sigma(0)}}{c_{i, \sigma(0)}-\gamma_{i} \bar{c}} & 0 & 0 \\
0 & 0 & 1 & 0 \\
0 & 0 & 0 & 1
\end{array}\right],
\end{aligned}
$$

and $c_{i, \sigma(0)}-\gamma_{i} \bar{c} \neq 0$. The optimal solution of equation (66) is

$$
\begin{aligned}
\bar{J}_{t}= & e_{\left(I-D_{1}\right) D_{1}^{-1}(1 / \mu)}(t, 0) \tilde{M}(t) \\
& -e_{\left(I-D_{1}\right) D_{1}^{-1}(1 / \mu)}(t, 0) \int_{0}^{T} e_{\Theta\left(I-D_{1}\right) D_{1}^{-1}(1 / \mu)}(s, 0) \frac{1}{\mu(s)} F_{1}(s) \Delta s,
\end{aligned}
$$


where $\bar{J}_{t}=\left[\begin{array}{c}\hat{a}_{i, t} \\ \widehat{c}_{i, t} \\ \hat{y}_{i, t} \\ \hat{r}_{t}\end{array}\right], \quad D_{1}=C_{1}^{-1} B_{1}, \quad F_{1}(t)=C_{1}^{-1}\left[\begin{array}{c}0 \\ \mu_{\sigma(t)}-\mu_{t} \\ 0 \\ 0\end{array}\right]$, and $\tilde{M}(t)$ is a $4 \times 1$ arbitrary matrix martingale and meets the martingale property $E_{t}\left[\widetilde{M}^{\sigma}(t)\right]=\tilde{M}(t)$.

Remark 5. When $\bar{c}=0$, utility function (35) becomes the CRRA utility function which describes the agents with constant relative risk aversion preferences. Equation (66) becomes a wealth distribution model with the CRRA utility function.

From solution (63) of model (36), when the initial values $a_{i, \sigma(0)}, c_{i, \sigma(0)}, y_{i, \sigma(0)}, \lambda_{i, \sigma(0)}, r_{\sigma(0)}, \bar{y}_{\sigma(0)}, \bar{a}_{\sigma(0)}, \bar{c}_{\sigma(0)}$, and $\bar{k}_{\sigma(0)}$ are fixed, the wealth distribution is only related to the parameters $\sigma_{i}, \gamma_{i}, \sigma, \phi_{i}, \varphi, \bar{\gamma}$, and $\bar{\phi}$, namely, model (36) depicts the influence of agent's trading behavior (the behavior of agent's risk aversion and the behavior of forecasting market risk and saving tendency) on wealth distribution.

\section{A Numerical Example}

To explain solution (63), we choose parameter values from $[21,28]$ to calculate it. We set $\gamma_{i}=0.9, \bar{\lambda}=0.5, \delta_{i}=0.2$, $\delta=0.5, \quad c_{i, 0}=c_{i, \sigma(0)}, \quad \bar{c}_{0}=\bar{c}_{\sigma(0)}=16, \quad a_{i, 0}=a_{i, \sigma(0)}=50$, $\bar{a}_{0}=\bar{a}_{\sigma(0)}=30, \quad k_{i, \sigma(0)}=0.98, \quad \bar{a}_{\sigma(0)}=0.32, \quad \lambda_{i, \sigma(0)}=0.9$, $r_{\sigma(0)}=0.2, \bar{\lambda}=0.6, \varphi=0.05, \phi_{i}=0.85, \bar{\phi}=0.7, y_{i, \sigma(0)}=20$, and $\bar{y}_{\sigma(0)}=15$. Then, the matrices $B, C$, and $D$ are obtained:

$$
\begin{aligned}
& B=\left[\begin{array}{ccccccc}
1.27 & 0 & 0 & 0 & 0 & 0 & 0 \\
0 & 5.86 & 0 & 0 & 0 & 0 & 0 \\
0 & 0 & 0.31 & -0.11 & 0 & 0 & 0 \\
0 & 0 & 0.625 & -0.125 & 0 & 0 & 0 \\
0 & 0 & 0 & 0 & 1 & 0 & 0 \\
0 & 0 & 0 & 0 & 0 & 1 & 0 \\
0 & 0 & 0 & 0 & 0 & 0 & 1
\end{array}\right] \\
& C=\left[\begin{array}{ccccccc}
1.25 & 0 & -1 & 0 & 0.51 & 0 & 0.133 \\
0 & 1.875 & 0 & -1 & 0 & 1.17 & 1.04 \\
0 & 0 & 0.3125 & -0.1125 & 0 & 0 & 0.174 \\
0 & 0 & 0.625 & -0.125 & 0 & 0 & 0.116 \\
0 & 0 & 0 & 0 & 0.85 & 0 & 0 \\
0 & 0 & 0 & 0 & 0 & 0.07 & 0 \\
0 & 0 & 0 & 0 & 0 & 0 & 0.95
\end{array}\right] \text {, } \\
& D=\left[\begin{array}{ccccccc}
1.0160 & 0 & 0.8080 & -0.0080 & -0.4800 & 0 & 0.1224 \\
0 & 3.1253 & 0.0267 & 0.5067 & 0 & -8.9143 & 0.7186 \\
0 & 0 & 1.0100 & -0.0100 & 0 & 0 & 0.2931 \\
0 & 0 & 0.0500 & 0.9500 & 0 & 0 & 2.4421 \\
0 & 0 & 0 & 0 & 1.1765 & 0 & 0 \\
0 & 0 & 0 & 0 & 0 & 14.2857 & 0 \\
0 & 0 & 0 & 0 & 0 & 0 & 1.0526
\end{array}\right]
\end{aligned}
$$

The Putzer algorithm on time scales [34] is employed to calculate the matrix $e_{(I-D) D^{-1}(1 / \mu)}(t, 0)$. Consequently, solution (63) is rewritten as 


$$
\begin{aligned}
& \widehat{a}_{i, t}=d_{11} M_{1}(t)+d_{12} M_{2}(t)+d_{13} M_{3}(t)+d_{14} M_{4}(t)+d_{15} M_{5}(t)+d_{16} M_{6}(t) \\
& +d_{17} M_{7}(t)-d_{13} \sum_{t \in[0, T) \mathbb{T}} g_{32}\left(\mu_{\sigma(t)}-\mu_{t}\right)-d_{14} \sum_{t \in[0, T) \mathbb{T}} g_{42}\left(\mu_{\sigma(t)}-\mu_{t}\right) \text {, } \\
& \widehat{\bar{a}}_{t}=d_{21} M_{1}(t)+d_{22} M_{2}(t)+d_{23} M_{3}(t)+d_{24} M_{4}(t)+d_{25} M_{5}(t)+d_{26} M_{6}(t) \\
& +d_{27} M_{7}(t)-d_{23} \sum_{t \in[0, T) \mathbb{T}} g_{32}\left(\mu_{\sigma(t)}-\mu_{t}\right)-d_{24} \sum_{t \in[0, T) \mathbb{T}} g_{42}\left(\mu_{\sigma(t)}-\mu_{t}\right), \\
& \widehat{c}_{i, t}=d_{31} M_{1}(t)+d_{32} M_{2}(t)+d_{33} M_{3}(t)+d_{34} M_{4}(t)+d_{35} M_{5}(t)+d_{36} M_{6}(t) \\
& +d_{37} M_{7}(t)-d_{33} \sum_{t \in[0, T) \mathbb{T}} g_{32}\left(\mu_{\sigma(t)}-\mu_{t}\right)-d_{34} \sum_{t \in[0, T) \mathbb{T}} g_{42}\left(\mu_{\sigma(t)}-\mu_{t}\right), \\
& \widehat{\bar{c}}_{t}=d_{41} M_{1}(t)+d_{42} M_{2}(t)+d_{43} M_{3}(t)+d_{44} M_{4}(t)+d_{45} M_{5}(t)+d_{46} M_{6}(t) \\
& +d_{47} M_{7}(t)-d_{13} \sum_{t \in[0, T) \mathbb{T}} g_{42}\left(\mu_{\sigma(t)}-\mu_{t}\right)-d_{44} \sum_{t \in[0, T) \mathbb{T}} g_{42}\left(\mu_{\sigma(t)}-\mu_{t}\right), \\
& \widehat{y}_{i, t}=d_{51} M_{1}(t)+d_{52} M_{2}(t)+d_{53} M_{3}(t)+d_{54} M_{4}(t)+d_{55} M_{5}(t)+d_{56} M_{6}(t) \\
& +d_{57} M_{7}(t)-d_{53} \sum_{t \in[0, T) \mathbb{T}} g_{42}\left(\mu_{\sigma(t)}-\mu_{t}\right)-d_{54} \sum_{t \in[0, T) \mathbb{T}} g_{42}\left(\mu_{\sigma(t)}-\mu_{t}\right) \text {, } \\
& \widehat{\bar{y}}_{t}=d_{61} M_{1}(t)+d_{62} M_{2}(t)+d_{63} M_{3}(t)+d_{64} M_{4}(t)+d_{65} M_{5}(t)+d_{66} M_{6}(t) \\
& +d_{67} M_{7}(t)-d_{63} \sum_{t \in[0, T) \mathbb{T}} g_{42}\left(\mu_{\sigma(t)}-\mu_{t}\right)-d_{64} \sum_{t \in[0, T) \mathbb{T}} g_{42}\left(\mu_{\sigma(t)}-\mu_{t}\right), \\
& \widehat{r}_{t}=d_{71} M_{1}(t)+d_{72} M_{2}(t)+d_{73} M_{3}(t)+d_{74} M_{4}(t)+d_{75} M_{5}(t)+d_{76} M_{6}(t) \\
& +d_{77} M_{7}(t)-d_{73} \sum_{t \in[0, T) \mathbb{T}} g_{42}\left(\mu_{\sigma(t)}-\mu_{t}\right)-d_{74} \sum_{t \in[0, T) \mathbb{T}} g_{42}\left(\mu_{\sigma(t)}-\mu_{t}\right),
\end{aligned}
$$

where $M_{1}(t), M_{2}(t), M_{3}(t), M_{4}(t), M_{5}(t), M_{6}(t)$, and $M_{7}(t)$ are arbitrary martingales and

$$
\begin{aligned}
d_{11}= & \lambda_{1}^{n_{t}} \\
d_{12}= & 0 \\
d_{16}= & 0 \\
d_{13}= & -66.49 \lambda_{1}^{n_{t}}-3.69 \times 10^{-3} \lambda_{2}^{n_{t}}-3.83 \lambda_{4}^{n_{t}}-2.77 \times 10^{-15} \lambda_{5}^{n_{t}} \\
& -8.39 \times 10^{-24} \lambda_{6}^{n_{t}}+6.74 \times 10^{-18} \lambda_{7}^{n_{t}}+70.33 \\
d_{14}= & 10.23 \lambda_{1}^{n_{t}}+3.64 \times 10^{-3} \lambda_{2}^{n_{t}}+3.84 \lambda_{4}^{n_{t}}+9.57 \times 10^{-17} \lambda_{5}^{n_{t}}-2.50 \times 10^{-23} \lambda_{6}^{n_{t}}-2.081 \times 10^{-18} \lambda_{7}^{n_{t}}-14.07, \\
d_{15}= & -10.82 \lambda_{1}^{n_{t}}+0.05 \lambda_{2}^{n_{t}}+0.001 \lambda_{4}^{n_{4}}+4.06 \lambda_{5}^{n_{t}}+1.37 \times 10^{-23} \lambda_{6}^{n_{t}}+1.7 \times 10^{-17} \lambda_{7}^{n_{t}}+6.701, \\
d_{17}= & -253.22 \lambda_{1}^{n_{t}}-0.12 \lambda_{2}^{n_{t}}-220.75 \lambda_{4}^{n_{t}}-13.13 \lambda_{5}^{n_{t}}+3.301 \times 10^{-7} \lambda_{6}^{n_{t}}+0.031 \lambda_{7}^{n_{t}}+1365.98, \\
d_{21}= & 0, \\
d_{22}= & \lambda_{2}^{n_{t}}-1.913 \times 10^{-5} \lambda_{1}^{n_{t}}, \\
d_{25}= & 0, \\
d_{23}= & -0.04 \lambda_{1}^{n_{t}}+0.017 \lambda_{2}^{n_{t}}-0.33 \lambda_{4}^{n_{t}}-1.22 \times 10^{-13} \lambda_{5}^{n_{t}}+2.11 \times 10^{-19} \lambda_{6}^{n_{t}} \\
& +3.15 \times 10^{-16} \lambda_{7}^{n_{t}}+0.35 \\
d_{24}= & -0.49 \lambda_{1}^{n_{t}}+0.23 \lambda_{2}^{n_{t}}+0.33 \lambda_{4}^{n_{t}}-2.87 \times 10^{-14} \lambda_{5}^{n_{t}}+4.95 \times 10^{-20} \lambda_{6}^{n_{t}} \\
& +7.43 \times 10^{-17} \lambda_{7}^{n_{t}}-0.07
\end{aligned}
$$




$$
\begin{aligned}
& d_{26}=13081293.87 \lambda_{1}^{n_{t}}+26451.40 \lambda_{2}^{n_{t}}+4751069.56 \lambda_{4}^{n_{t}}+336.031 \lambda_{5}^{n_{t}} \\
& -28057.56 \lambda_{6}^{n_{t}}-9.65 \times 10^{-10} \lambda_{7}^{n_{t}}-17831521.75 \text {, } \\
& d_{27}=27.45 \lambda_{1}^{n_{t}}+0.63 \lambda_{2}^{n_{t}}+27.55 \lambda_{4}^{n_{t}}+3.51 \lambda_{5}^{n_{t}}-8.83 \times 10^{-8} \lambda_{6}^{n_{t}} \\
& -0.008 \lambda_{7}^{n_{t}}-294.10 \text {, } \\
& d_{31}=0 \text {, } \\
& d_{32}=0 \text {, } \\
& d_{35}=0 \text {, } \\
& d_{36}=0 \text {, } \\
& d_{33}=2.13 \lambda_{1}^{n_{t}}+0.000265 \lambda_{2}^{n_{t}}+0.28 \lambda_{4}^{n_{t}}+2.44 \times 10^{-17} \lambda_{5}^{n_{t}}-1.58 \times 10^{-24} \lambda_{6}^{n_{t}} \\
& -578 \lambda_{7}^{n_{t}}-1.41 \text {, } \\
& d_{34}=-0.0013 \lambda_{1}^{n_{t}}-0.00026 \lambda_{2}^{n_{t}}-0.28 \lambda_{4}^{n_{t}}+5.02 \times 10^{-17} \lambda_{5}^{n_{t}}+2.21 \times 10^{-24} \lambda_{6}^{n_{t}} \\
& -1.24 \times 10^{-19} \lambda_{7}^{n_{t}}+0.28 \\
& d_{37}=-5.26 \lambda_{1}^{n_{t}}+0.0044 \lambda_{2}^{n_{t}}+0.022 \lambda_{4}^{n_{t}}-0.64 \lambda_{5}^{n_{t}}+1.62 \times 10^{-8} \lambda_{6}^{n_{t}} \\
& +0.0015 \lambda_{7}^{n_{t}}+49.02 \text {, } \\
& d_{41}=0 \text {, } \\
& d_{42}=0 \text {, } \\
& d_{45}=0 \text {, } \\
& d_{46}=0 \text {, } \\
& d_{43}=0.00694 \lambda_{1}^{n_{t}}+0.00132 \lambda_{2}^{n_{t}}+1.4 \lambda_{4}^{n_{t}}-2.49 \times 10^{-16} \lambda_{5}^{n_{t}}-1.1 \times 10^{-23} \lambda_{6}^{n_{t}}+6.15 \times 10^{-19} \lambda_{7}^{n_{t}}-1.41, \\
& d_{44}=2.12 \lambda_{1}^{n_{t}}-0.00132 \lambda_{2}^{n_{t}}-1.4 \lambda_{4}^{n_{t}}+3.24 \times 10^{-16} \lambda_{5}^{n_{t}}+1.16 \times 10^{-23} \lambda_{6}^{n_{t}}-7.96 \times 10^{-19} \lambda_{7}^{n_{t}}+0.281 \text {, } \\
& d_{47}=-111.0 \lambda_{1}^{n_{t}}-0.00503 \lambda_{2}^{n_{t}}-103.0 \lambda_{4}^{n_{t}}-13.5 \lambda_{5}^{n_{t}}+3.4 \times 10^{-7} \lambda_{6}^{n_{t}} 0.0322 \lambda_{7}^{n_{t}}+1133.0, \\
& d_{51}=0 \text {, } \\
& d_{52}=0 \text {, } \\
& d_{53}=0 \text {, } \\
& d_{54}=0 \text {, } \\
& d_{56}=0 \text {, } \\
& d_{57}=0 \text {, } \\
& d_{55}=4.62 \lambda_{1}^{n_{t}}-0.0177 \lambda_{2}^{n_{t}}-3.84 \times 10^{-4} \lambda_{4}^{n_{t}}-1.36 \lambda_{5}^{n_{t}}-2.24, \\
& d_{61}=0 \text {, } \\
& d_{62}=0 \text {, } \\
& d_{63}=0 \text {, } \\
& d_{64}=0 \text {, } \\
& d_{65}=0 \text {, } \\
& d_{67}=0 \text {, } \\
& d_{66}=-1.64 \times 10^{7} \lambda_{1}^{n_{t}}-3.31 \times 10^{4} \lambda_{2}^{n_{t}}-5.95 \times 10^{6} \lambda_{4}^{n_{t}}-421.0 \lambda_{5}^{n_{t}}+3.51 \times 10^{4} \lambda_{6}^{n_{t}}+2.23 \times 10^{7},
\end{aligned}
$$




$$
\begin{aligned}
& d_{71}=0 \\
& d_{72}=0 \\
& d_{73}=0 \\
& d_{74}=0 \\
& d_{75}=0 \\
& d_{76}=0 \\
& d_{77}=-2.43 \lambda_{1}^{n_{t}}-0.00147 \lambda_{2}^{n_{t}}-5.57 \lambda_{4}^{n_{t}}-0.556 \lambda_{5}^{n_{t}}+1.4 \times 10^{-8} \lambda_{6}^{n_{t}}+0.00132 \lambda_{7}^{n_{t}}+46.8, \\
& g_{32}=1.35 \times 10^{6} \lambda_{a}^{n_{t}}-3.44 \lambda_{b}^{n_{t}}+1.56 \times 10^{5} \lambda_{d}^{n_{t}}-1177.0 \lambda_{e}^{n_{t}}+2.76 \times 10^{-4} \lambda_{f}^{n_{t}}-3.34 \times 10^{5} \lambda_{g}^{n_{t}}-1.21 \times 10^{6}, \\
& g_{42}=1.35 \times 10^{6} \lambda_{a}^{n_{t}}-3.44 \lambda_{b}^{n_{t}}+1.56 \times 10^{5} \lambda_{d}^{n_{t}}-1177.0 \lambda_{e}^{n_{t}}+2.76 \times 10^{-4} \lambda_{f}^{n_{t}}-3.34 \times 10^{5} \lambda_{g}^{n_{t}}-1.21 \times 10^{6},
\end{aligned}
$$

with the eigenvalues of matrix $D^{-1}$ and $D$ as $\lambda_{1}=1.016$, $\lambda_{2}=3.1253, \lambda_{3}=1, \lambda_{4}=0.96, \lambda_{5}=1.1765, \lambda_{6}=14.2857$, and $\lambda_{7}=1.0526$ and $\lambda_{a}=0.9843, \lambda_{b}=0.32, \quad \lambda_{c}=1$, $\lambda_{d}=1.0417, \quad \lambda_{e}=0.85, \quad \lambda_{f}=0.07, \quad$ and $\quad \lambda_{a}=0.95$, respectively.

From the calculation process of solution (63), the effect of isolated discrete time domains on model (34) is obvious. When $\mathbb{T}=\mathbb{Z}$, we get

$$
\left\{\begin{array}{l}
a_{i, t}=a_{i, 0}\left(1+\sum_{i=1}^{7} d_{1 i} M_{i}(t)\right), \\
c_{i, t}=c_{i, 0}\left(1+\sum_{i=1}^{7} d_{3 i} M_{i}(t)\right) .
\end{array}\right.
$$

It is worth noting that system (72) is a closed form optimal solution for model (34). System (72) explains the distribution of wealth among agents as the trading time interval is uniform. The risk aversion parameter $\delta_{i}$ depicts the influence of agent's risk aversion on wealth distribution. $\phi$ and $\varphi$ describe the distribution of wealth among agents as the agents predict market risks.

\section{Conclusion}

In this paper, a wealth distribution model on isolated discrete time domains is given to describe the process of wealth distribution. We assume that agents have different degrees of risk aversion. The HARA utility function is employed to characterize the degrees of risk aversion of agents, including decreasing relative risk aversion, increasing relative risk aversion, and constant relative risk aversion. We consider the influence of agent's expectation and average consumption level on wealth distribution. In addition, we allow agents to adopt certain trading strategies to maximize their utility and improve their wealth status. The Euler equation and transversality condition of the nonlinear stochastic dynamic model on isolated discrete time domains are given to prove the existence of the optimal solution of the model. The optimal solution of the wealth distribution model is obtained by using the method of solving the rational expectation model on isolated discrete time domains. A numerical example is given to illustrate the advantages of the wealth distribution model on isolated discrete time domains. The main advantage of the wealth distribution model is that, unlike the wealth distribution models in which wealth exchange is assumed to take place at evenly spaced intervals, the intervals between wealth exchange are of any arbitrary length.

\section{Appendix}

\section{A. The Derivation of Equation (44)}

Log-linearizing $y_{i, \sigma(t)} /\left(2 \lambda+\lambda r_{\sigma(t)}-1\right)$, we get

$$
\begin{aligned}
& \frac{1}{2 \lambda+} \lambda r_{\sigma(t)}-1 \\
& y_{i, \sigma(t)} \\
& \approx \frac{1}{2 \lambda+\lambda r_{\sigma(0)}-1} y_{i, \sigma(0)}+\frac{\left(y_{i, \sigma(t)}-y_{i, \sigma(0)}\right)}{2 \lambda+\lambda r_{\sigma(0)}-1} \\
&-\frac{\lambda y_{i, \sigma(0)}}{\left(2 \lambda+\lambda r_{\sigma(0)}-1\right)^{2}}\left(r_{\sigma(t)}-r_{\sigma(0)}\right) \\
& \approx \frac{1}{2 \lambda+\lambda r_{\sigma(0)}-1} y_{i, \sigma(0)}+\frac{y_{i, \sigma(0)}}{2 \lambda+\lambda r_{\sigma(0)}-1} \\
& \times \frac{y_{i, \sigma(t)}-y_{i, \sigma(0)}}{y_{i, \sigma(0)}} \frac{\lambda y_{i, \sigma(0)} r_{\sigma(0)}}{\left(2 \lambda+\lambda r_{\sigma(0)}-1\right)^{2}} \frac{r_{\sigma(t)}-r_{\sigma(0)}}{r_{\sigma(0)}} \\
& \approx \frac{1}{2 \lambda+\lambda r_{\sigma(0)}-1} y_{i, \sigma(0)}+\frac{y_{i, \sigma(0)}}{2 \lambda+\lambda r_{\sigma(0)}-1} \widehat{y}_{i, \sigma(t)} \\
&-\frac{\lambda y_{i, \sigma(0)} r_{\sigma(0)}}{\left(2 \lambda+\lambda r_{\sigma(0)}-1\right)^{2}} \widehat{r}_{\sigma(t)} .
\end{aligned}
$$


Similarly, log-linearizing the first and second terms on the right side of equation (41a), we obtain

$$
\begin{aligned}
& a_{i, t}-\frac{1}{2 \lambda+\lambda r_{\sigma(t)}-1} a_{i, \sigma(t)} \\
& \quad \approx a_{i, 0}\left(1+\widehat{a}_{i, t}\right)-\frac{a_{i, 0}}{2 \lambda+\lambda r_{\sigma(0)}-1}-\frac{1}{2 \lambda+\lambda r_{\sigma(0)}-1}\left(a_{i, \sigma(t)}-a_{i, \sigma(0)}\right)+\frac{\lambda a_{i, \sigma(0)}}{\left(2 \lambda+\lambda r_{\sigma(0)}-1\right)^{2}}\left(r_{\sigma(t)}-r_{\sigma(0)}\right) .
\end{aligned}
$$

Thus, we have

$$
\begin{aligned}
a_{i, t} & -\frac{1}{2 \lambda+\lambda r_{\sigma(t)}-1} a_{i, \sigma(t)} \\
& \approx a_{i, 0}\left(1+\widehat{a}_{i, t}\right)-\frac{a_{i, 0}}{2 \lambda+\lambda r_{\sigma(0)}-1}-\frac{a_{i, \sigma(0)}}{2 \lambda+\lambda r_{\sigma(0)}-1} \frac{a_{i, \sigma(t)}-a_{i, \sigma(0}}{a_{i, \sigma(0)}}+\frac{\lambda a_{i, \sigma(0)} r_{\sigma(0)}}{\left(2 \lambda+\lambda r_{\sigma(0)}-1\right)^{2}} \frac{r_{\sigma(t)}-r_{\sigma(0}}{r_{\sigma(0)}} \\
& \approx a_{i, 0}\left(1+\widehat{a}_{i, t}\right)-\frac{a_{i, \sigma(0)}}{2 \lambda+\lambda r_{\sigma(0)}-1}-\frac{a_{i, \sigma(0)}}{2 \lambda+\lambda r_{\sigma(0)}-1} \widehat{a}_{i, \sigma(t)}+\frac{\lambda a_{i, \sigma(0)} r_{\sigma(0)}}{\left(2 \lambda+\lambda r_{\sigma(0)}-1\right)^{2}} \widehat{r}_{\sigma(t)} .
\end{aligned}
$$

Combining equations (A.1) and (A.3), we get

$$
\begin{aligned}
c_{i, t} \approx & c_{i, 0}+c_{i, 0} \widehat{c}_{i, t} \\
\approx & a_{i, 0}\left(1+\widehat{a}_{i, t}\right)-\frac{a_{i, \sigma(0)}}{2 \lambda+\lambda r_{\sigma(0)}-1}-\frac{a_{i, \sigma(0)} \widehat{a}_{i, \sigma(t)}}{2 \lambda+\lambda r_{\sigma(0)}-1}+\frac{\lambda a_{i, \sigma(0)} r_{\sigma(0)}}{\left(2 \lambda+\lambda r_{\sigma(0)}-1\right)^{2}} \widehat{r}_{\sigma(t)}+\frac{y_{i, \sigma(0)}}{2 \lambda+\lambda r_{\sigma(0)}-1} \\
& +\frac{y_{i, \sigma(0)}}{2 \lambda+\lambda r_{\sigma(0)}-1} \widehat{y}_{i, \sigma(t)}-\frac{\lambda y_{i, \sigma(0)} r_{\sigma(0)}}{\left(2 \lambda+\lambda r_{\sigma(0)}-1\right)^{2}} \widehat{r}_{\sigma(t)} \\
\approx & a_{i, 0}-\frac{a_{i, \sigma(0)}}{2 \lambda+\lambda r_{\sigma(0)}-1}+\frac{y_{i, \sigma(0)}}{2 \lambda+\lambda r_{\sigma(0)}-1}+a_{i, 0} \widehat{a}_{i, t}+\frac{1}{2 \lambda+\lambda r_{\sigma(0)}-1}\left(y_{i, \sigma(0)} \widehat{y}_{i, \sigma(t)}-a_{i, \sigma(0)} \widehat{a}_{i, \sigma(t)}\right) \\
& +\frac{\lambda r_{\sigma(0)}}{\left(2 \lambda+\lambda r_{\sigma(0)}-1\right)^{2}}\left(a_{i, \sigma(0)} \widehat{r}_{\sigma(t)}-y_{i, \sigma(0)} \widehat{r}_{\sigma(t)}\right) .
\end{aligned}
$$

Based on initial state (42a), equation (A.4) becomes

$$
\begin{aligned}
c_{i, t} \approx & c_{i, 0}+a_{i, 0} \widehat{a}_{i, t}+\frac{y_{i, \sigma(0)} \widehat{y}_{i, \sigma(t)}-a_{i, \sigma(0)} \widehat{a}_{i, \sigma(t)}}{2 \lambda+\lambda r_{\sigma(0)}-1} \\
& +\frac{\lambda r_{\sigma(0)} \widehat{r}_{\sigma(t)}}{\left(2 \lambda+\lambda r_{\sigma(0)}-1\right)^{2}}\left(a_{i, \sigma(0)}-y_{i, \sigma(0)}\right) \widehat{r}_{\sigma(t)} .
\end{aligned}
$$

Thus, we obtain

$$
\begin{aligned}
c_{i, 0} \widehat{c}_{i, t} \approx & a_{i, 0} \widehat{a}_{i, t}+\frac{y_{i, \sigma(0)} \hat{y}_{i, \sigma(t)}-a_{i, \sigma(0)} \hat{a}_{i, \sigma(t)}}{2 \lambda+\lambda r_{\sigma(0)}-1} \\
& +\frac{\lambda r_{\sigma(0)}}{\left(2 \lambda+\lambda r_{\sigma(0)}-1\right)^{2}}\left(a_{i, \sigma(0)}-y_{i, \sigma(0)}\right) \widehat{r}_{\sigma(t)} .
\end{aligned}
$$

\section{B. The Derivation of Equation (48)}

For system (39), we log-linearize the first equation. That is, 


$$
\begin{aligned}
\beta_{i} \frac{\mu_{\sigma(t)}}{\mu_{t}}\left(\frac{c_{i, t}-\gamma_{i} \bar{c}_{t}}{c_{i, \sigma(t)}-\gamma_{i} \bar{c}_{\sigma(t)}}\right)^{\delta_{i}} k_{\sigma(t)} \\
\approx \beta_{i} \frac{\mu_{\sigma(0)}}{\mu_{0}}\left(\frac{c_{i, 0}-\gamma_{i} \bar{c}_{0}}{c_{i, \sigma(0)}-\gamma_{i} \bar{c}_{\sigma(0)}}\right)^{\delta_{i}} k_{\sigma(0)} \\
\quad+\frac{\beta_{i}}{\mu_{0}}\left(\frac{c_{i, 0}-\gamma_{i} \bar{c}_{0}}{c_{i, \sigma(0)}-\gamma_{i} \bar{c}_{\sigma(0)}}\right)^{\delta_{i}} k_{\sigma(0)}\left(\mu_{\sigma(t)}-\mu_{\sigma(0)}\right) \\
\quad-\beta_{i} \frac{\mu_{\sigma(0)}}{\mu_{0}^{2}}\left(\frac{c_{i, 0}-\gamma_{i} \bar{c}_{0}}{c_{i, \sigma(0)}-\gamma_{i} \bar{c}_{\sigma(0)}}\right)^{\delta_{i}} k_{\sigma(0)}\left(\mu_{t}-\mu_{0}\right) \\
\quad+\beta_{i} \frac{\mu_{\sigma(0)}}{\mu_{0}} \frac{\left(c_{i, 0}-\gamma_{i} \bar{c}_{0}\right)^{\delta_{i}-1}}{\left(c_{i, \sigma(0)}-\gamma_{i} \bar{c}_{\sigma(0)}\right)^{\delta_{i}}} \delta_{i} k_{\sigma(0)}\left(c_{i, t}-c_{i, 0}\right) \\
\left.\quad-\gamma_{i} \beta_{i} \frac{\mu_{\sigma(0)}}{\mu_{0}} \frac{\left(c_{i, 0}-\gamma_{i} \bar{c}_{0}\right)^{\delta_{i}-1}}{\left(c_{i, \sigma(0)}-\gamma_{i} \bar{c}_{\sigma(0)}\right.}\right)^{\delta_{i}} \delta_{i} k_{\sigma(0)}\left(\bar{c}_{t}-\bar{c}_{0}\right) \\
\quad-\delta_{i} \beta_{i} \frac{\mu_{\sigma(0)}}{\mu_{0}} \frac{\left(c_{i, 0}-\gamma_{i} \bar{c}_{0}\right)^{\delta_{i}}}{\left(c_{i, \sigma(0)}-\gamma_{i} \bar{c}_{\sigma(0)}\right)^{\delta_{i}+1}} k_{\sigma(0)}\left(c_{i, \sigma(t)}-c_{i, \sigma(0)}\right) \\
+\frac{\mu_{\sigma(0)}}{\mu_{0}} \frac{\left(c_{i, 0}-\gamma_{i} \bar{c}_{0}\right)^{\delta_{i}}}{\left(c_{i, \sigma(0)}-\gamma_{i} \bar{c}_{\sigma(0)}\right)^{\delta_{i}+1}} \delta_{i} \gamma_{i} \beta_{i} k_{\sigma(0)}\left(\bar{c}_{\sigma(t)}-\bar{c}_{\sigma(0)}\right) \\
+\frac{\beta_{i} \mu_{\sigma(0)}}{\mu_{0}}\left(\frac{c_{i, 0}-\gamma_{i} \bar{c}_{0}}{c_{i, \sigma(0)}-\gamma_{i} \bar{c}_{\sigma(0)}}\right)^{\delta_{i}}\left(k_{\sigma(t)}-k_{\sigma(0)}\right) .
\end{aligned}
$$

Since $\hat{x}_{t}=\left(x_{t}-x_{0}\right) / x_{0}$, equation (B.1) is turned into

$$
\begin{aligned}
& \beta_{i} \frac{\mu_{\sigma(t)}}{\mu_{t}}\left(\frac{c_{i, t}-\gamma_{i} \bar{c}_{t}}{c_{i, \sigma(t)}-\gamma_{i} \bar{c}_{\sigma(t)}}\right)^{\delta_{i}} k_{\sigma(t)} \\
& \approx \beta_{i} \frac{\mu_{\sigma(0)}}{\mu_{0}}\left(\frac{c_{i, 0}-\gamma_{i} \bar{c}_{0}}{c_{i, \sigma(0)}-\gamma_{i} \bar{c}_{\sigma(0)}}\right)^{\delta_{i}} k_{\sigma(0)} \\
& +\frac{\beta_{i} \mu_{\sigma(0)}}{\mu_{0}}\left(\frac{c_{i, 0}-\gamma_{i} \bar{c}_{0}}{c_{i, \sigma(0)}-\gamma_{i} \bar{c}_{\sigma(0)}}\right)^{\delta_{i}} k_{\sigma(0)} \mu_{\sigma(t)} \\
& -\beta_{i} \frac{\mu_{\sigma(0)}}{\mu_{0}}\left(\frac{c_{i, 0}-\gamma_{i} \bar{c}_{0}}{c_{i, \sigma(0)}-\gamma_{i} \bar{c}_{\sigma(0)}}\right)^{\delta_{i}} k_{\sigma(0)} \mu_{t} \\
& +\beta_{i} \frac{\mu_{\sigma(0)} c_{i, 0}}{\mu_{0}} \frac{\left(c_{i, 0}-\gamma_{i} \bar{c}_{0}\right)^{\delta_{i}-1}}{\left(c_{i, \sigma(0)}-\gamma_{i} \bar{c}_{\sigma(0)}\right)^{\delta_{i}}} \delta_{i} k_{i, \sigma(0)} \widehat{c}_{i, t} \\
& -\gamma_{i} \beta_{i} \frac{\mu_{\sigma(0)} \bar{c}_{0}}{\mu_{0}} \frac{\left(c_{i, 0}-\gamma_{i} \bar{c}_{0}\right)^{\delta_{i}-1}}{\left(c_{i, \sigma(0)}-\gamma_{i} \bar{c}_{\sigma(0)}\right)^{\delta_{i}}} \delta_{i} k_{\sigma(0)} \hat{\bar{c}}_{t} \\
& -\delta_{i} \beta_{i} k_{\sigma(0)} \frac{\mu_{\sigma(0)} c_{i, \sigma(0)}}{\mu_{0}} \frac{\left(c_{i, 0}-\gamma_{i} \bar{c}_{0}\right)^{\delta_{i}}}{\left(c_{i, \sigma(0)}-\gamma_{i} \bar{c}_{\sigma(0)}\right)^{\delta_{i}+1}} \widehat{c}_{i, \sigma(t)} \\
& +\delta_{i} \gamma_{i} \beta_{i} k_{\sigma(0)} \frac{\mu_{\sigma(0)}}{\mu_{0}} \frac{\left(c_{i, 0}-\gamma_{i} \bar{c}_{0}\right)^{\delta_{i}}}{\left(c_{i, \sigma(0)}-\gamma_{i} \bar{c}_{\sigma(0)}\right)^{\delta_{i}+1}} \bar{c}_{\sigma(0)} \hat{\bar{c}}_{\sigma(t)} \\
& +\frac{\beta_{i} \mu_{\sigma(0)}}{\mu_{0}}\left(\frac{c_{i, 0}-\gamma_{i} \bar{c}_{0}}{c_{i, \sigma(0)}-\gamma_{i} \bar{c}_{\sigma(0)}}\right)^{\delta_{i}} k_{\sigma(0)} \widehat{k}_{\sigma(t)} .
\end{aligned}
$$

By elementary algebra, we get

$$
\begin{aligned}
& \beta_{i} \frac{\mu_{\sigma(t)}}{\mu_{t}}\left(\frac{c_{i, t}-\gamma_{i} \bar{c}_{t}}{c_{i, \sigma(t)}-\gamma_{i} \bar{c}_{\sigma(t)}}\right)^{\delta_{i}} k_{\sigma(t)} \\
& \approx \frac{\beta_{i} \mu_{\sigma(0)}}{\mu_{0}}\left(\frac{c_{i, 0}-\gamma_{i} \bar{c}_{0}}{c_{i, \sigma(0)}-\gamma_{i} \bar{c}_{\sigma(0)}}\right)^{\delta_{i}} k_{\sigma(0)}\left[1+\mu_{\sigma(t)}-\mu_{t}\right. \\
& +\frac{c_{i, 0} \delta_{i}}{c_{i, 0}-\gamma_{i} \bar{c}_{0}} \widehat{c}_{i, t}-\frac{\delta_{i} \bar{c}_{0} \gamma_{i}}{c_{i, 0}-\gamma_{i} \bar{c}_{0}}-\frac{c_{i, \sigma(0)} \delta_{i}}{c_{i, \sigma(0)}-\gamma_{i} \bar{c}_{\sigma(0)}} \widehat{c}_{i, \sigma(t)} \\
& \left.+\frac{\delta_{i} \gamma_{i} \bar{c}_{\sigma(0)}}{c_{i, \sigma(0)}-\gamma_{i} \bar{c}_{\sigma(0)}} \hat{\bar{c}}_{\sigma(t)}+\widehat{k}_{\sigma(t)}\right] .
\end{aligned}
$$

On the basis of equations (42d) and (B.3), we get equation (48). Similarly, we log-linearize the second equation in system (39) and obtain equation (49).

\section{The Derivation of Equation (50)}

To log-linearize equation (41d), we rewrite it at $\sigma(t)$ and obtain

$$
\log y_{i, \sigma(t)}=\left(1-\phi_{i}\right) \log y_{i, 0}+\phi_{i} \log y_{i, t}+\varepsilon_{i, \sigma(t)} .
$$

Log-linearizing on both sides of equation (C.1), we get

$$
\begin{array}{r}
\log y_{i, \sigma(0)}+\frac{1}{y_{i, \sigma(0)}}\left(y_{i, \sigma(t)}-y_{i, \sigma(0)}\right) \approx\left(1-\phi_{i}\right) \log y_{i, 0}+\phi_{i} \log y_{i, 0} \\
+\phi_{i} \frac{1}{y_{i, 0}}\left(y_{i, t}-y_{i, 0}\right)+\varepsilon_{i, \sigma(t)}, \\
\log y_{i, \sigma(0)}+\hat{y}_{i, \sigma(t)} \approx\left(1-\phi_{i}\right) \log y_{i, 0}+\phi_{i} \log y_{i, 0} \\
+\phi_{i} \hat{y}_{i, t}+\varepsilon_{i, \sigma(t)} .
\end{array}
$$

Since $\log y_{i, \sigma(0)}=\left(1-\phi_{i}\right) \log y_{i, 0}+\phi_{i} \log y_{i, 0}+\varepsilon_{i, \sigma(0)}$, we obtain equation (50).

\section{The Derivation of Equation (54)}

To obtain the log-linearization expression of equation (4lf), we write equation (41f) as follows:

$$
\log r_{\sigma(t)}=\varphi \log r_{0}+(1-\varphi) \log r_{t}+\eta_{\sigma(t)} .
$$

Log-linearizing equation (D.1), we obtain

$$
\begin{aligned}
\log r_{\sigma(0)}+\frac{1}{r_{\sigma(0)}}\left(r_{\sigma(t)}-r_{\sigma(0)}\right) \approx & \varphi \log r_{0}+(1-\varphi) \log r_{0} \\
& +(1-\varphi) \frac{1}{r_{0}}\left(r_{t}-r_{0}\right)+\eta_{\sigma(t)},
\end{aligned}
$$

$\log r_{\sigma(0)}+\widehat{r}_{\sigma(t)} \approx \varphi \log r_{0}+(1-\varphi) \log r_{0}+(1-\varphi) \widehat{r}_{t}+\eta_{\sigma(t)}$. 
Since $\log r_{\sigma(0)}=\varphi \log r_{0}+(1-\varphi) \log r_{0}+\eta_{\sigma(0)}$, equation (D.3) becomes equation (54).

\section{Data Availability}

No data were used to support this study.

\section{Conflicts of Interest}

No potential conflicts of interest have been reported by the authors.

\section{Acknowledgments}

This research was supported by the National Natural Science Foundation of China (no. 11471263).

\section{References}

[1] S. Guala, "Taxes in a wealth distribution model by inelastically scattering of particles," Interdisciplinary Description of Complex Systems, vol. 7, no. 1, pp. 1-7, 2009.

[2] L. Pareschi and G. Toscani, "Wealth distribution and collective knowledge: a Boltzmann approach," Philosophical Transactions: Mathematical, Physical and Engineering Sciences, vol. 372, no. 2028, pp. 1-16, 2014.

[3] G. Toscani, C. Brugna, and S. Demichelis, "Kinetic models for the trading of goods," Journal of Statistical Physics, vol. 151, no. 3-4, pp. 549-566, 2013.

[4] C. Brugna and G. Toscani, "Kinetic models for goods exchange in a multi-agent market," Physica A: Statistical Mechanics and Its Applications, vol. 499, pp. 362-375, 2018.

[5] A. S. Chakrabarti and B. K. Chakrabarti, "Microeconomics of the ideal gas like market models," Physica A: Statistical Mechanics and Its Applications, vol. 388, no. 19, pp. 4151-4158, 2009.

[6] A. S. Chakrabarti and B. K. Chakrabarti, "Inequality reversal: effects of the savings propensity and correlated returns," Physica A: Statistical Mechanics and Its Applications, vol. 389, no. 17, pp. 3572-3579, 2010.

[7] A. S. Chakrabarti, "Scale-free distribution as an economic invariant: a theoretical approach," Journal of Economic Interaction and Coordination, vol. 12, no. 1, pp. 1-26, 2017.

[8] F. Clementi, M. Gallegati, G. Kaniadakis, and S. Landini, " $\kappa$-generalized models of income and wealth distributions: a survey," The European Physical Journal Special Topics, vol. 225, no. 10, pp. 1959-1984, 2016.

[9] A. Chatterjee, B. K. Chakrabarti, and S. S. Manna, "Pareto law in a kinetic model of market with random saving propensity," Physica A: Statistical Mechanics and Its Applications, vol. 335, no. 1-2, pp. 155-163, 2004.

[10] J. Benhabib, A. Bisin, and S. Zhu, "The wealth distribution in Bewley economies with capital income risk," Journal of Economic Theory, vol. 159, pp. 489-515, 2015.

[11] D. N. Mariacristina and F. Giulio, "Saving and wealth inequality," Review of Economic Dynamics, vol. 26, pp. 280-300, 2017.

[12] A. Chatterjee and B. K. Chakrabarti, "Kinetic exchange models for income and wealth distributions," The European Physical Journal B, vol. 60, no. 2, pp. 135-149, 2007.
[13] A. S. Chakrabarti, "Bimodality in the firm size distributions: a kinetic exchange model approach," European Physical Journal B, vol. 86, no. 6, pp. 255-261, 2013.

[14] L. Blake, "Econophysics of agent-based models," Journal of Economic Literature, vol. 52, no. 3, pp. 855-858, 2014.

[15] A. Ghosh, U. Basu, A. Chakraborti, and B. K. Chakrabarti, "Threshold-induced phase transition in kinetic exchange models," Physical Review E, vol. 83, no. 6, pp. 61130-61136, 2011.

[16] S. Goswami and A. Chakraborti, "Kinetic exchange models in economics and sociology," Nonlinear Maps and Their Applications, vol. 112, pp. 69-88, 2015.

[17] J. Acedńnski, "Heterogeneous expectations and the distribution of wealth," Journal of Macroeconomics, vol. 53, pp. 162-175, 2017.

[18] F. M. Atici, D. C. Biles, and A. Lebedinsky, "An application of time scales to economics," Mathematical and Computer Modelling, vol. 43, no. 7-8, pp. 718-726, 2006.

[19] F. M. Atici, D. C. Biles, and A. Lebedinsky, "A utility maximisation problem on multiple time scales," International Journal of Dynamical Systems and Differential Equations, vol. 3, no. 1-2, pp. 38-47, 2011.

[20] F. M. Atici and N. Turhan, "Sequential decision problems on isolated time domains," Journal of Mathematical Analysis and Applications, vol. 388, no. 2, pp. 753-759, 2012.

[21] F. M. Atici, G. Cheng, and A. Lebedinsky, "A nonlinear stochastic growth model on discrete time domains," Journal of Difference Equations and Applications, vol. 22, no. 11, pp. 1732-1746, 2016.

[22] J. Li, H. Hugh, and H. T. Liu, "Minimum system sensitivity study of linear discrete time systems for fault detection," Mathematical Problems in Engineering, vol. 2013, Article ID 276987, 13 pages, 2013.

[23] Y. Zheng, C. Li, and Z. Zhao, "A fully discrete discontinuous galerkin method for nonlinear fractional Fokker-Planck equation," Mathematical Problems in Engineering, vol. 2010, pp. 1-26, 2010.

[24] F. M. Atici, F. Ekiz, and A. Lebedinsky, "Cagan type rational expectation model on complex discrete time domains," $E \mathbf{U}$ ropean Journal of Operational Research, vol. 237, no. 1, pp. 148-151, 2014.

[25] C. Hu, S. Lai, and Z. Dou, "On the study of a rational expectation model with lagged endogenous variables," Journal of Difference Equations and Applications, vol. 25, no. 3, pp. 351-372, 2019.

[26] C. Achury, S. Hubar, and C. Koulovatianos, "Saving rates and portfolio choice with subsistence consumption," Review of Economic Dynamics, vol. 15, no. 1, pp. 108-126, 2012.

[27] R. Levaggi and F. Menoncin, "Optimal dynamic tax evasion," Journal of Economic Dynamics and Control, vol. 37, no. 11, pp. 157-167, 2013.

[28] F. Menoncin and S. Nembrini, "Stochastic continuous time growth models that allow for closed form solutions," Journal of Economics, vol. 124, no. 3, pp. 213-241, 2018.

[29] E. Merrell, R. Ruger, and J. Severs, "First order recurrence relations on isolated time scales," Panamerican Mathematical Journal, vol. 14, no. 1, pp. 83-104, 2004.

[30] B. G. Jia, L. Erbe, and A. Peterson, "A Butler-type oscillation theorem for second-order dynamic equations on discrete time scales," Journal of Difference Equations and Applications, vol. 20, no. 5-6, pp. 671-684, 2014.

[31] M. Bohner, "Calculus of variations on time scales," Dynamic Systems and Applications, vol. 13, no. 3-4, pp. 339-349, 2004. 
[32] N. Stokey and R. Lucas, Recursive Methods in Economic Dynamics, Harvard University Press, Cambridge, UK, 1989.

[33] F. C. Klebaner, Introduction to Stoachastic Calculus with Application, Imperial College Press, London, UK, 2005.

[34] M. Bohner and A. Peterson, "Dynamic equations on time scaless," in An Introduction with Applications, Birkhäuser, Boston, MA, USA, 2001. 